\title{
ON ALMOST-FIXED-POINT THEORY
}

\author{
MICHIEL HAZEWINKEL AND MARCEL VAN DE VEL
}

1. Introduction. Let $X$ be a topological space, $\mathscr{U}$ a finite covering of $X$ (the words 'covering' and 'cover' are used interchangeably). We say that $(X, \mathscr{U})$ has the almost fixed point property for a class $\mathscr{F}$ of continuous maps $f: X \rightarrow X$ if for all $f \in \mathscr{F}$ there is an $x \in X$ and $U \in \mathscr{U}$ such that $x \in U$ and $f(x) \in U$, or, equivalently, if there is a $U \in \mathscr{U}$ such that $U \cap f(U) \neq \emptyset$.

For example, if $X$ is the euclidean plane and $\mathscr{U}$ a finite open covering by convex sets, then $(X, \mathscr{U})$ has the almost fixed point property for all continuous maps (cf. De Groot, De Vries, Van der Walt [3]). Other examples of almost fixed point theorems can be found in Klee [8], Halpern [7] and Gray, Vaughan $[6]$.

It is fairly natural to restrict attention to finite coverings of $X$. Indeed if a Hausdorff space $X$ is such that $(X, \mathscr{U})$ has the almost fixed point property for all open coverings $\mathscr{U}$, then $X$ has the fixed point property. It is also fairly natural to concentrate somewhat on noncompact spaces $X$ because if a compact Hausdorff space $X$ is such that $(X, \mathscr{Z})$ has the almost fixed point property for all (or a cofinal set of all) finite coverings $\mathscr{U}$ then $X$ has the fixed point property.

There is an extension of this last result. Let $X$ be a $T_{4}$-space and $f: X \rightarrow X$ a continuous map. Let $w(X)$ be the Wallman compactification of $X$. There is an induced continuous map $w(f): w(X) \rightarrow w(X)$. The following two statements are then equivalent: (i) $w(f)$ has a fixed point; (ii) for every finite open covering $\mathscr{U}$ of $X$ there is a $U \in \mathscr{U}$ such that $U \cap f(U) \neq \emptyset$. This follows from the fact that there is a one-one correspondence between finite coverings of $X$ and finite coverings of $w(X)$.

In this paper we develop what Thompson [13] calls an indirect theory. A main result is a Lefschetz-type almost fixed point theorem. We first define a certain kind of finite coverings called geometric coverings. The result then is: let $X$ be a space with a closed geometric covering $\mathscr{C}$ and let $f: X \rightarrow X$. Then $L(f)=0$ or for every finite open cover $\mathscr{U}$ which is refined by $\mathscr{C}$ there is a $U \in \mathscr{U}$ such that $U \cap f(U) \neq \emptyset$. Here $L(f)$, the Lefschetz number, is defined in terms of compactly generated Čech homology.

The next step is then to find at least some examples of geometric coverings. In this direction we have, for example, the following results:

(i) A compact space $X$ admits a weak semicomplex structure (cf. Thompson [12] for this notion) if and only if every finite open covering is geometric. (Spaces which admit a WSC structure include all compact polyhedra).

Received August 4, 1976 and in revised form, January 17, 1978. 


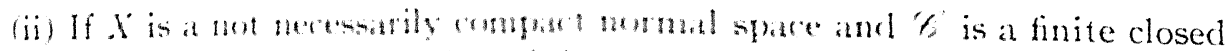

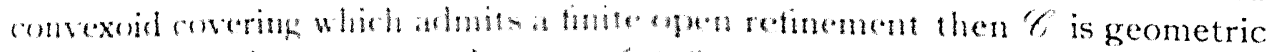

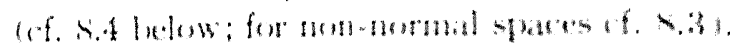

This last result, the Lefohelatye almost fixed print theorem, and a result on the existence of finile dened comsex refinements of tinite open convex

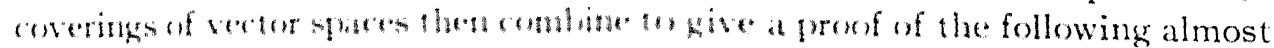

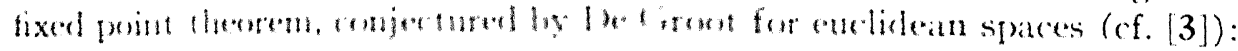

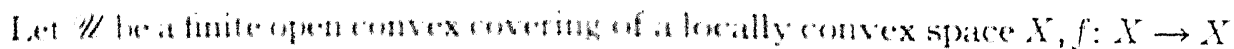

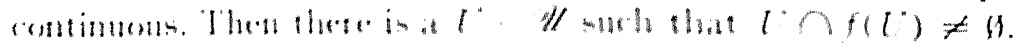

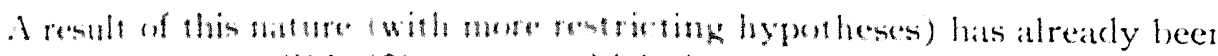

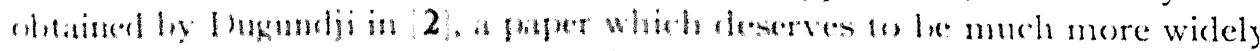

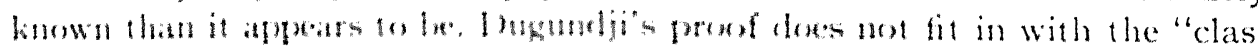
sical" procedure of lefoluete heerl gum theory, and his hypotheses ar homotopical, rather than homologiral.

2. Compactly generated Gech homology. In this section we introduc stme notation and give as shom cunline of the defintions of the (compactl

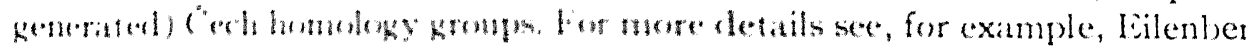
and Steromed 4 and Spronier 11

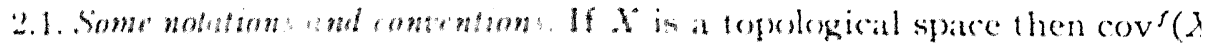

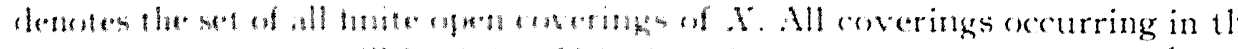

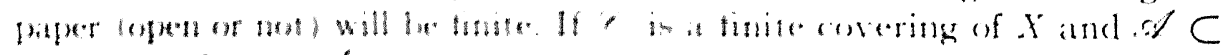

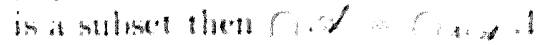

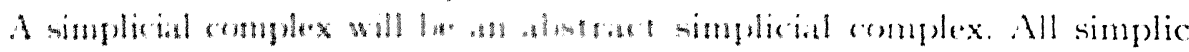

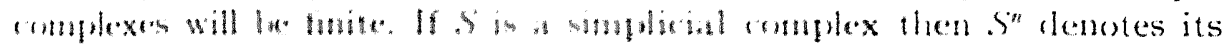

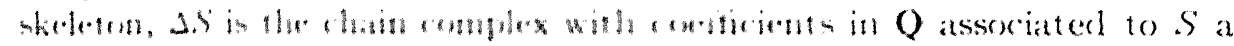

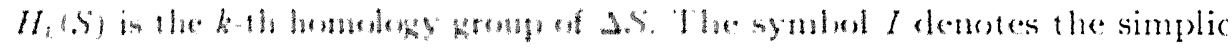

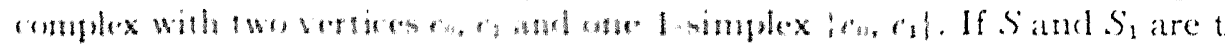

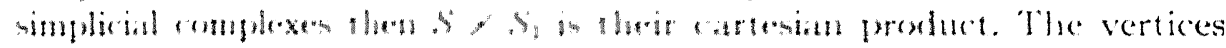

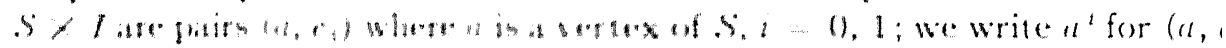

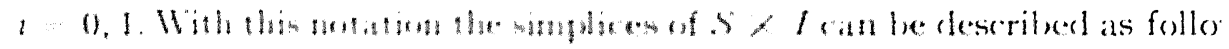

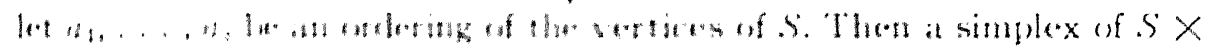

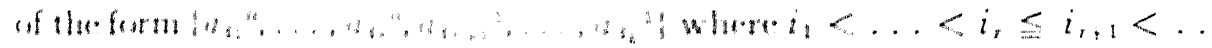

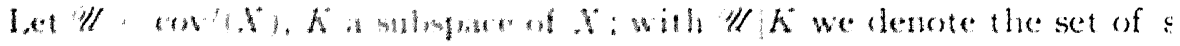

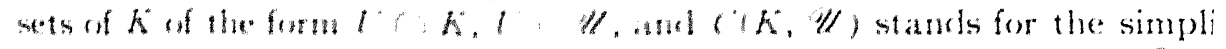

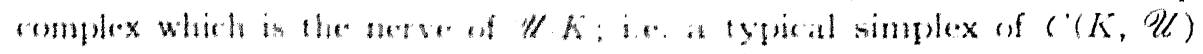

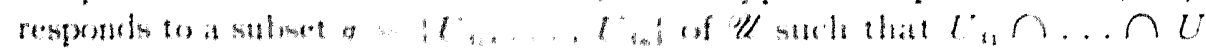

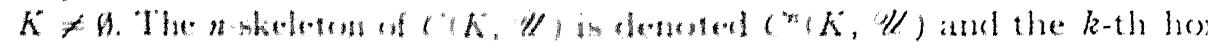

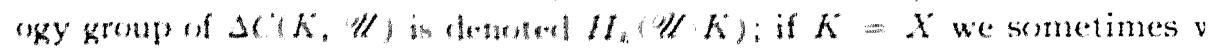

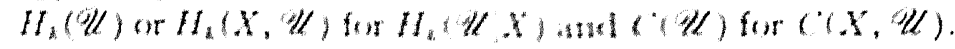


(ii) If $X$ is a not necessarily compact normal space and $\mathscr{C}$ is a finite clo: convexoid covering which admits a finite open refinement then $\mathscr{C}$ is geome (cf. 8.4 below; for non-normal spaces cf. 8.3).

This last result, the Lefschetz-type almost fixed point theorem, an result on the existence of finite closed convex refinements of finite open con coverings of vector spaces then combine to give a proof of the following aln fixed point theorem, conjectured by De Groot for euclidean spaces (cf. [3]

Let $\mathscr{U}$ be a finite open convex covering of a locally convex space $X, f: X$ continuous. Then there is a $U \in \mathscr{U}$ such that $U \cap f(U) \neq \emptyset$.

A result of this nature (with more restricting hypotheses) has already 1 obtained by Dugundji in [2], a paper which deserves to be much more wi known than it appears to be. Dugundji's proof does not fit in with the " sical" procedure of Lefschetz fixed point theory, and his hypotheses homotopical, rather than homological.

2. Compactly generated Čech homology. In this section we intrc some notation and give a short outline of the definitions of the (comp generated) Cech homology groups. For more details see, for example, Eiles and Steenrod [4] and Spanier [11].

2.1. Some notations and conventions. If $X$ is a topological space then cor denotes the set of all finite open coverings of $X$. All coverings occurring $i$ paper (open or not) will be finite. If $\mathscr{C}$ is a finite covering of $X$ and $\mathscr{A}$ is a subset then $\cap \mathscr{A}=\cap_{A \in \mathscr{A}} A$.

A simplicial complex will be an abstract simplicial complex. All sim complexes will be finite. If $S$ is a simplicial complex then $S^{n}$ denotes skeleton, $\Delta S$ is the chain complex with coefficients in $\mathbf{Q}$ associated to $H_{k}(S)$ is the $k$-th homology group of $\Delta S$. The symbol $I$ denotes the sim complex with two vertices $e_{0}, e_{1}$ and one 1 -simplex $\left\{e_{0}, e_{1}\right\}$. If $S$ and $S_{1}$ a simplicial complexes then $S \times S_{1}$ is their cartesian product. The vert $S \times I$ are pairs $\left(a, e_{i}\right)$ where $a$ is a vertex of $S, i=0,1$; we write $c^{i}$ for $i=0,1$. With this notation the simplices of $S \times I$ can be described as $\mathrm{ft}_{\mathrm{t}}$ let $a_{1}, \ldots, a_{s}$ be an ordering of the vertices of $S$. Then a simplex of $S$ of the form $\left\{a_{i_{1}}{ }^{0}, \ldots, a_{i_{r}}{ }^{0}, a_{i_{r+1}}{ }^{1}, \ldots, a_{i_{k}}{ }^{1}\right\}$ where $i_{1}<\ldots<i_{r} \leqq i_{r+1}<$

Let $\mathscr{U} \in \operatorname{cov}^{f}(X), K$ a subspace of $X$; with $\mathscr{U} \mid K$ we denote the set sets of $K$ of the form $U \cap K, U \in \mathscr{U}$, and $C(K, \mathscr{U})$ stands for the sir complex which is the nerve of $\mathscr{U} \mid K$; i.e. a typical simplex of $C(K, \zeta$ responds to a subset $\sigma=\left\{U_{i_{1}}, \ldots, U_{i_{n}}\right\}$ of $\mathscr{U}$ such that $U_{i_{1}} \cap \ldots \cap$ $K \neq \emptyset$. The $n$-skeleton of $C(K, \mathscr{U})$ is denoted $C^{n}(K, \mathscr{U})$ and the $k$-th ogy group of $\Delta C(K, \mathscr{U})$ is denoted $H_{k}(\mathscr{U} \mid K)$; if $K=X$ we sometim $H_{k}(\mathscr{U})$ or $H_{k}(X, \mathscr{U})$ for $H_{k}(\mathscr{U} \mid X)$ and $C(\mathscr{U})$ for $C(X, \mathscr{U})$. 
Let $\sigma$ be a simplex of $C(K, \mathscr{U}), \mathscr{U} \in \operatorname{cov}^{f}(X), K \subset X$. Then the support of $\sigma, \operatorname{supp}(\sigma)$, is defined as $\cup_{v \in \sigma} U \cap K$ and the reduced support of $\sigma$ as rsupp $(\sigma)$ $=\cap_{U \in \sigma} U \cap K$. A chain $c \in \Delta C(K, \mathscr{U})$ is said to be on a subspace $A \subset K$ if $c$ is in the subchain complex $\Delta C(A, \mathscr{U}) \subset \Delta C(K, \mathscr{U})$ or, equivalently, if $\operatorname{rsupp}(\sigma) \cap A \neq \emptyset$ for all $\sigma$ occurring in $c$ (i.e. having nonzero coefficient in $c$ ).

2.2. Čech homology of compact spaces. Let $K$ be a compact space, $K^{\prime}$ a closed (compact) subspace of $K$. Let $\mathscr{U}^{\prime} \in \operatorname{cov}^{f}\left(K^{\prime}\right)$ and $\mathscr{U} \in \operatorname{cov}^{f}(K)$. Then we say that $\mathscr{U}^{\prime}$ refines $\mathscr{U}$ and write $\mathscr{U}^{\prime} \leqq \mathscr{U}$ if for every $U^{\prime} \in \mathscr{U}^{\prime}$ there is a $U \in \mathscr{U}$ such that $U^{\prime} \subset U$. Choosing such a $V$ for every $V^{\prime} \in \mathscr{U}^{\prime}$ defines a map of simplicial complexes $C\left(K^{\prime}, \mathscr{U}^{\prime}\right) \rightarrow C(K, \mathscr{U})$ and a homomorphism of chain complexes $\Delta C\left(K^{\prime}, \mathscr{U}^{\prime}\right) \rightarrow \Delta C(K, \mathscr{U})$ and induces a homomorphism $H_{k}\left(\mathscr{U}^{\prime}\right) \rightarrow$ $H_{k}(\mathscr{U})$. All these maps are called refinement maps. There is usually more than one refinement map $C\left(K^{\prime}, \mathscr{U}^{\prime}\right) \rightarrow C(K, \mathscr{U})$ but they are all homotopic and hence induce the same homomorphism $H_{k}\left(\mathscr{U}^{\prime}\right) \rightarrow H_{k}(\mathscr{U})$.

Applying this with $K^{\prime}=K$ and letting $\mathscr{U}$ run through $\operatorname{cov}^{f}(K)$ we obtain a projective system of groups and homomorphisms, $H_{k}(K,-)$, indexed by $\operatorname{cov}^{f}(K)$. The $k$-th Čech homology group of $K$ is now defined as $H_{k}(K)=$ $\lim _{\leftarrow} H_{k}(K,-)$. The canonical projection $H_{k}(K) \rightarrow H_{r}(\mathscr{U})$ is denoted by $g_{\vartheta_{l}}{ }^{K}$.

Let $f: K_{1} \rightarrow K_{2}$ be a continuous map of compact spaces. Every $\mathscr{U}_{2} \in$ $\operatorname{cov}^{f}\left(K_{2}\right)$ then gives rise to a $f^{-1} \mathscr{U}_{2}=\mathscr{U}_{1} \in \operatorname{cov}^{f}\left(K_{1}\right), f^{-1}\left(\mathscr{U}_{2}\right)=\left\{f^{-1} U_{2} \mid U_{2} \in\right.$ $\left.\mathscr{U}_{2}\right\}$. Assigning to a vertex $f^{-1} U_{2}$ the vertex $U_{2}$ defines a map of simplicial complexes $C\left(K_{1}, \mathscr{U}_{1}\right) \rightarrow C\left(K_{2}, \mathscr{U}_{2}\right)$ and induces a homomorphism $H_{k}(f)$ : $H_{k}\left(\mathscr{U}_{1}\right) \rightarrow H_{k}\left(\mathscr{U}_{2}\right)$. Letting $\mathscr{U}_{2}$ run through $\operatorname{cov}^{f}\left(K_{2}\right)$ we obtain a homomorphism of projective systems $H_{k}(f): H_{k}\left(K_{1},-\right) \rightarrow H_{k}\left(K_{2},-\right)$ which in turn gives rise to a homomorphism $H_{k}(f): H_{k}\left(K_{1}\right) \rightarrow H_{k}\left(K_{2}\right)$.

Note that we have a commutative diagram

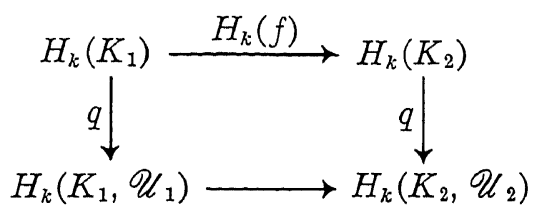

whenever $\mathscr{U}_{1}$ refines $f^{-1} \mathscr{U}_{2}$ (where the lower horizontal homomorphism in the composite of a refinement map $H_{k}\left(K_{1}, \mathscr{U}_{1}\right) \rightarrow H_{k}\left(K_{1}, f^{-1} \mathscr{U}_{2}\right)$ and $H_{k}(f)$ : $H_{k}\left(K_{1}, f^{-1} \mathscr{U}_{2}\right) \rightarrow H_{k}\left(K_{2}, \mathscr{U}_{2}\right)$.

The Čech homology theory on compact spaces (or more generally compact pairs) satisfies all the usual homology axioms, cf. Eilenberg and Steenrod [4].

2.3. Compactly generated Čech homology. Now let $X$ be a not necessarily compact topological space. We could, of course, again write down the definitions of 2.2 and thus define Čech homology groups of $X$ based on finite covers. However, this homology theory does not satisfy the homotopy axiom (e.g. the 
Cech homology based on finite covers of $\mathbf{R}$ is not trivial). Instead we take compactly generated Čech homology, also called Čech homology with compact supports, which is defined as follows.

Let $\Gamma$ be a cofinal collection of compact subsets of $X$, i.e. for every compact $K \subset X$, there is a $K^{\prime} \in \Gamma$ such that $K \subset K^{\prime}$. For each $K \in \Gamma$, write down $H_{k}(K)$ : if $K_{1} \subset K_{2}, K_{1}, K_{2} \in \Gamma$ we have an induced homomorphism $H_{k}\left(K_{1}\right) \rightarrow$ $H_{k}\left(K_{2}\right)$ giving us an injective system of homology groups indexed by $\Gamma$. We now define

$$
H_{k}(X)=\lim _{K \in \Gamma} H_{k}(K) .
$$

This definition does not depend on $\Gamma$. If $f: X \rightarrow Y$ is a continuous map then for every $K \subset X, K$ compact, we have that $f(K) \subset Y$ is compact and hence we have an induced map $H_{k}(K) \rightarrow H_{k}(f K)$. This gives us a homomorphism of inductive systems and, taking the limit, an induced homomorphism $H_{k}(f): H_{k}(X) \rightarrow H_{k}(Y)$.

For compact spaces $X$ these definitions agree with the ones from 2.2.

Let $\mathscr{U} \in \operatorname{cov}^{f}(X)$, i.e. $\mathscr{U}$ is a finite open cover of the (not necessarily compact) space $X$. Then there is a natural homomorphism $q_{\mathscr{U}}{ }^{X}: H_{k}(X) \rightarrow H_{k}(\mathscr{U})$ which is defined as follows. Let $z \in H_{k}(X)$, then there is a compact $K$ and a $z^{\prime} \in H_{k}(K)$ such that $z^{\prime}$ is mapped onto $z$ under the natural homomorphism $H_{k}(K) \rightarrow H_{k}(X)$. Enlarging $K$ if necessary we can assume that $C(K, \mathscr{U})=$ $C(X, \mathscr{U})$. We now define $q_{\mathscr{U}}{ }^{X}(z)=q_{\mathscr{U}^{K}}{ }^{\prime}\left(z^{\prime}\right)$. This does not depend on $K$.

Let $f: X \rightarrow Y$ be a continuous map, $\mathscr{V} \in \operatorname{cov}^{f}(Y), \mathscr{U} \in \operatorname{cov}^{f}(X)$ and suppose that $\mathscr{U} \leqq f^{-1 \mathscr{V}}$. Then we have a commutative diagram

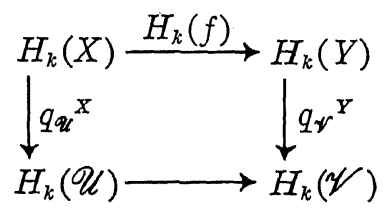

where the lower horizontal homomorphism is defined in the obvious way.

2.4. Reduced homology groups. The chain complexes $\Delta C(K, \mathscr{U})$ carry a natural augmentation. The homology groups of the augmented complex are the reduced homology groups denoted $\tilde{H}_{k}(K, \mathscr{U})$. Replacing $H_{k}(K, \mathscr{U})$ with $\widetilde{H}_{k}(K, \mathscr{U})$ everywhere in 2.2 and 2.3 then defines reduced Čech homology groups $\widetilde{H}_{k}(X), \widetilde{H}_{k}(K)$.

2.5. Lefschetz' theorem. Let $X$ be a compact space, $\mathscr{U} \in \operatorname{cov}^{f}(X)$. Then there is a $\mathscr{V} \in \operatorname{cov}^{f}(X)$ which refines $\mathscr{U}$ such that

$$
\operatorname{Im}(H(X) \rightarrow H(\mathscr{U}))=\operatorname{Im}(H(\mathscr{V}) \rightarrow H(\mathscr{U})) .
$$

This follows directly from the fact that the $H(\mathscr{U})$ are finite dimensional vector spaces over $\mathbf{Q}$. The same result holds for reduced homology (for the same reason). 
3. Geometric covers. In this section we define and discuss the main technical tool of this paper, the notion of what we like to call a geometric cover.

3.1. Definition of geometric covers. Let $X$ be a topological space. A finite (not necessarily open) cover $\mathscr{C}$ of $X$ is geometric in dimensions $\leqq n$ with respect to compactly generated Čech homology if there exist

(i) a cofinal collection $\Gamma$ of compact subsets of $X$;

(ii) a map $\gamma: \Gamma \rightarrow \Gamma$ such that

(3.1.1) $K \subset \gamma(K)$ for all $K \in \Gamma$;

(iii) a finite open refinement $\mathscr{U}^{\prime}$ of $\mathscr{C}$;

(iv) for every $K \in \Gamma$ and $\mathscr{V} \in \operatorname{cov}^{f}(\gamma K)$ such that $\mathscr{V} \leqq \mathscr{U}^{\prime}$ an augmentation preserving chain map $\tau_{\mathscr{V}}: \Delta C^{n+1}\left(K, \mathscr{U}^{\prime}\right) \rightarrow \Delta C(\gamma K, \mathscr{V})$ such that the following conditions are satisfied:

(3.1.2) (Factorization property). If $k \leqq n, K \in \Gamma, \mathscr{V} \in \operatorname{cov}^{f}(\gamma K), \mathscr{V} \leqq$ $\mathscr{U}^{\prime}$, then there is a $\mathscr{V}^{\prime} \in \operatorname{cov}^{f}(K), \mathscr{V}^{\prime} \leqq \mathscr{V}$ such that the following diagram commutes (where the two unlabelled arrows are induced by refinement maps).

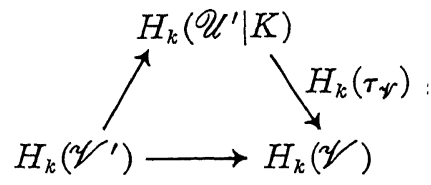

(3.1.3) (Compatibility property). If $k \leqq n, K \in \Gamma, \mathscr{V}_{1}, \mathscr{V}_{2} \in \operatorname{cov}^{f}(\gamma K)$, $\mathscr{V}_{1} \leqq \mathscr{V}_{2} \leqq \mathscr{U}^{\prime}$, then the following diagram commutes:

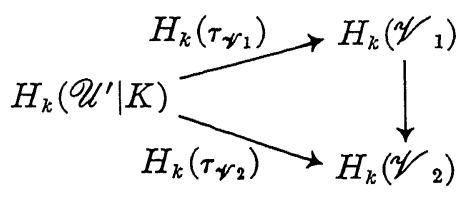

(3.1.4) (Norm condition). For every $\mathscr{V}$ and for every $\sigma \in C^{n+1}\left(K, \mathscr{U}^{\prime}\right)$ there is a $C \in \mathscr{C}$ such that $\operatorname{rsupp}(\sigma) \subset C \cap K$ and $\tau_{\mathscr{V}}(\sigma)$ is on $C \cap \gamma K$; i.e. $\operatorname{rsupp}\left(\sigma^{\prime}\right) \cap C \cap \gamma K \neq \emptyset$ for all $\sigma^{\prime}$ occurring in $\tau_{\mathscr{V}}(\sigma)$.

In the sequel we shall use $n$-geometric as an abbreviation for: "geometric in dimensions $\leqq n$ with respect to compactly generated Čech homology."

A finite cover $\mathscr{C}$ is geometric if there exists a finite open refinement $\mathscr{U}^{\prime} \in$ $\operatorname{cov}^{f}(X)$ of $\mathscr{C}$ such that there are, for every $n \in \mathbf{N}$, a cofinal collection of compact sets $\Gamma_{n}$, a map $\gamma_{n}: \Gamma_{n} \rightarrow \Gamma_{n}$, and for every $K_{n} \in \Gamma_{n}$ and $\mathscr{V} \in \operatorname{cov}^{\mathcal{f}}\left(\gamma_{n} K_{n}\right)$, $\mathscr{V} \leqq \mathscr{U}^{\prime}$ chain maps $\tau_{\mathscr{V}}^{(n)}: \Delta C^{n+1}\left(K_{n}, \mathscr{U}^{\prime}\right) \rightarrow \Delta C\left(\gamma_{n} K_{n}, \mathscr{V}\right)$ such that (3.1.1)-(3.1.4) hold. Note that $\mathscr{U}^{\prime}$ is not allowed to depend on $n$ (but that everything else may depend on $n$ ). 
Examples of geometric covers are all finite open covers of compact spaces which admit a weak semicomplex structure (cf. 3.2 and 3.3 below).

If $X$ is a not necessarily compact normal space and $\mathscr{C}$ is a finite closed convexoid cover which has a finite open refinement, then $\mathscr{C}$ is geometric (cf. 8.4).

As in the case of weak semicomplexes the existence of geometric covers is closely related to various forms of local acyclicity (or local connectedness) of the space $X$ (cf. 4.5 and Sections 7 and 8).

Let $\mathscr{C}$ be an $n$-geometric (or geometric) cover of a space $X$. Then $\mathfrak{U}(\mathscr{C})$ denotes the collection of all covers $\mathscr{U}^{\prime}$ of $X$ refining $\mathscr{C}$ such that there exist corresponding $\Gamma, \gamma, \tau_{\mathscr{\gamma}}$ satisfying the conditions listed above.

Note that $\mathscr{U}^{\prime} \in \mathfrak{Q}(\mathscr{C})$ and $\mathscr{U}^{\prime \prime} \in \operatorname{cov}^{f}(X), \mathscr{U}^{\prime \prime} \leqq \mathscr{U}^{\prime} \Rightarrow \mathscr{U}^{\prime \prime} \in \mathfrak{U}(\mathscr{C})$ and $\mathscr{U}^{\prime} \in \mathfrak{I}(\mathscr{C}), \mathscr{C} \leqq \mathscr{C}^{\prime} \Rightarrow \mathscr{U}^{\prime} \in \mathfrak{U}\left(\mathscr{C}^{\prime}\right)$, i.e. every cover of $X$ refined by an $n$-geometric one is itself $n$-geometric.

3.2. Weak semicomplex structures (Thompson $[\mathbf{1 2}]$ ). Let $X$ be a compact space. A reak semicomplex structure (WSC) on $X$ consists of:

(i) for every $\mathscr{U} \in \operatorname{cov}^{f}(X)$ a cofinal subset $\Omega(\mathscr{U}) \subset \operatorname{cov}^{f}(X)$ with a coarsest element $\alpha(\mathscr{U}) \in \Omega(\mathscr{U}), \alpha(\mathscr{U}) \leqq \mathscr{U}$;

(ii) for every $\mathscr{V}, \mathscr{W} \in \Omega(\mathscr{U}), \mathscr{V} \leqq \mathscr{W}$ an augmentation preserving chain map $c_{\mathscr{V}}{ }^{\mathscr{W}}: \Delta C(X, \mathscr{W}) \rightarrow \Delta C(X, \mathscr{V})$ such that the following conditions are satisfied:

(3.2.1) If $\mathscr{V}^{\prime \prime} \leqq \mathscr{V}^{\prime} \leqq \mathscr{V}$ in $\Omega(\mathscr{U})$ then the following diagrams are commutative up to homotopy (where the unlabelled arrows are refinement maps)
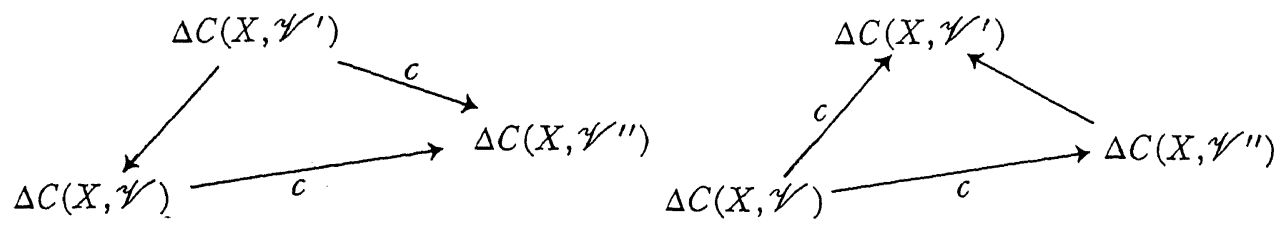

(3.2.2) For each $\mathscr{V} \in \Omega(\mathscr{U}), c_{\mathscr{r}}{ }^{\mathscr{V}}$ induces an idempotent homomorphism $H(\mathscr{V}) \rightarrow H(\mathscr{V})$ of which the image coincides with the image of $H(X)$ in $H(\mathscr{V})$ (under the natural map).

(3.2.3) If $\mathscr{V} \leqq \mathscr{W}$ in $\Omega(\mathscr{U})$ then the chain maps $c_{\mathscr{W}}{ }^{W}$ satisfy the following norm condition: For every $c_{\mathscr{\psi}}^{\mathscr{W}}: \Delta C(X, \mathscr{W}) \rightarrow \Delta C(X, \mathscr{V})$ and every simplex $\sigma \in C(X, \mathscr{W})$ there exists a $U \in \mathscr{U}$ such that $\operatorname{supp}(\sigma) \subset U$ and $\operatorname{supp}\left(c_{\mathscr{\gamma}}{ }^{\mathscr{W}}(\sigma)\right) \subset U$.

3.3. TheOREm. Let $X$ be a compact space which admits aWSC structure. Then every finite open cover of $X$ is geometric.

Proof. Let $\mathscr{U} \in \operatorname{cov}^{f}(X)$. Take $\mathscr{U}^{\prime}=\alpha(\mathscr{U})$ (cf. 3.2 above). We take $\Gamma=\{X\}$, and define $\tau_{\mathscr{V}}: C\left(X, \mathscr{U}^{\prime}\right) \rightarrow \Delta C(\mathrm{X}, \mathscr{V})$ for every $\mathscr{V} \in \operatorname{cov}^{f}(X)$ refining $\mathscr{U}^{\prime}$ as follows: 
If $\mathscr{V} \in \Omega(\mathscr{U})$ take $\tau_{\mathscr{V}}=c_{\mathscr{\Upsilon}}{ }^{\mathscr{\prime}}$.

If $\mathscr{V} \notin \Omega(\mathscr{U})$ let $\mathscr{V}^{\prime} \in \Omega(\mathscr{U})$ be a refinement of $\mathscr{V}$ and define $\tau_{\mathscr{V}}$ as the composite of $c_{\mathscr{V}^{\prime}}{ }^{\mathscr{\prime}^{\prime}}$ with the refinement map $\Delta C\left(X, \mathscr{V}^{\prime}\right) \rightarrow \Delta C(X, \mathscr{V})$.

This definition does not depend on $\mathscr{V}^{\prime}$ (up to homotopy) because of (3.2.1) (second diagram).

We check the various axioms. (3.1.1) is automatic and (3.1.3) and (3.1.4) follow from respectively (3.2.1) (second diagram) and (3.2.3). It remains to prove the factorization condition. Again it suffices to do this for $\mathscr{V} \in \Omega(\mathscr{U})$. Let $\mathscr{V}^{\prime} \leqq \mathscr{V}$ be such that the image of $H\left(\mathscr{V}^{\prime}\right)$ in $H(\mathscr{V})$ is equal to the image of $H(X)$ in $H(\mathscr{V})$. Such a $\mathscr{V} \in \operatorname{cov}^{f}(X)$ exists by the Lefschetz theorem 2.5. We have a diagram

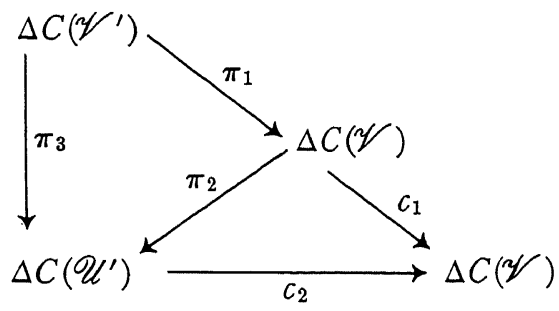

and this diagram is commutative up to homotopy. Now according to (3.2.2) $c_{1}$ * is idempotent with as image the image of $H(X)$ in $H(\mathscr{V})$.

It follows that $c_{1 *} \pi_{1 *}=\pi_{1} *$. And hence we have $\pi_{1 *}=c_{1 * \pi_{1} *}=c_{2} * \pi_{2} * \pi_{1} *=$ $c_{2} * \pi_{3} *$ which proves the factorization.

Conversely we have the following.

3.4. THEOREM. Let $X$ be a compact space and suppose that every open covering of $X$ is geometric. Then $X$ admits a WSC structure.

Proof. Let $\mathscr{U}_{0} \in \operatorname{cov}^{s}(X)$, let $\mathscr{U}$ be a starrefinement of $\mathscr{U}_{0}$. The cover $\mathscr{U}$ is geometric by hypothesis; let $\mathscr{U}^{\prime} \in \mathfrak{H}(\mathscr{U})$. We take $\alpha\left(\mathscr{U}_{0}\right)=\mathscr{U}^{\prime}$ and $\Omega\left(\mathscr{U}_{0}\right)=$ $\left\{\mathscr{V} \in \operatorname{cov}^{f}(X) \mid \mathscr{V} \leqq \mathscr{U}^{\prime}\right\}$. Let $\Gamma, \gamma, \tau_{\mathscr{V}}$ be the other structure elements which make $\mathscr{U}$ a geometric cover. Taking $X=K \in \Gamma$ we have augmentation preserving chain maps

$$
\tau_{\mathscr{V}}: C\left(\mathscr{U}^{\prime}\right) \rightarrow \Delta C(\mathscr{V})
$$

for all $\mathscr{V} \in \Omega\left(\mathscr{U}_{0}\right)$ satisfying (3.1.2)-(3.1.4). For $\mathscr{V} \leqq \mathscr{W} \leqq \mathscr{U}^{\prime}$ we define the inverse projections $c_{\mathscr{V}}{ }^{\mathscr{V}}: C(\mathscr{W}) \rightarrow \Delta C(\mathscr{V})$ as the composite of a refinement map $C(\mathscr{W}) \rightarrow C\left(\mathscr{U}^{\prime}\right)$ with $\tau_{\mathscr{V}}: C\left(\mathscr{U}^{\prime}\right) \rightarrow \Delta C(\mathscr{V})$. It is now not difficult to check the commutativity up to homotopy of the diagrams (3.2.1). Indeed if $\mathscr{V}_{3} \leqq \mathscr{V}_{2} \leqq \mathscr{V}_{1} \leqq \mathscr{U}^{\prime}$ we have the diagrams

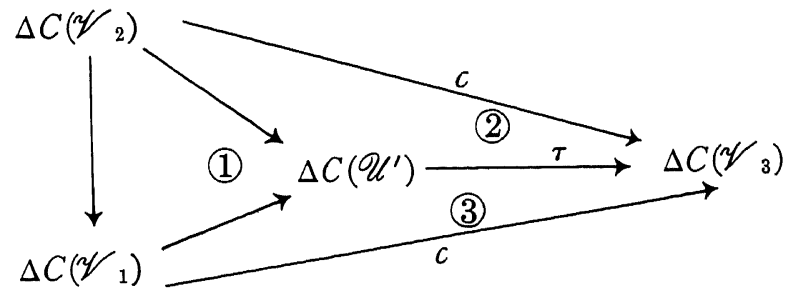




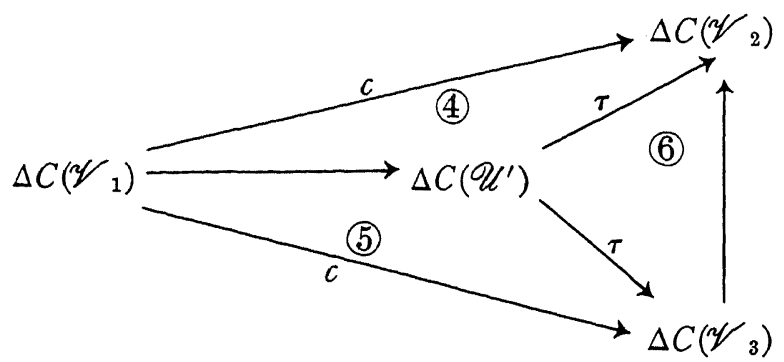

where the unlabelled arrows are refinement maps. The triangles $1,2,3,4,5,6$ are commutative up to homotopy because of respectively: triangle of refinement maps; definition $c$; definition $c$; definition $c$; definition $c$; compatibility. The outer triangles are therefore commutative which is what we needed to prove.

We now check the norm condition. Let $\mathscr{V} \leqq \mathscr{W}, c: \Delta C(\mathscr{W}) \rightarrow \Delta C(\mathscr{V})$ the inverse projection. The map $c$ is defined as the composite of a refinement map $\pi: \Delta C(\mathscr{W}) \rightarrow \Delta C\left(\mathscr{U}^{\prime}\right)$ and $\tau_{\mathscr{V}}: \Delta C\left(\mathscr{Q}^{\prime}\right) \rightarrow \Delta C(\mathscr{V})$. Let $\sigma$ be a simplex of $\Delta C(\mathscr{W})$; then $\pi \sigma$ is a simplex of $\Delta C\left(\mathscr{U}^{\prime}\right)$. Hence because of the norm condition (3.1.4) there is a $U \in \mathscr{U}$ such that

$$
\operatorname{rsupp}(\pi \sigma) \subset U \text { and } \tau(\pi \sigma) \text { is on } U \text {. }
$$

Because $\mathscr{U}$ is a starrefinement of $\mathscr{U}_{0}$, this implies that there is $U_{0} \in \mathscr{U}_{0}$ such that $\operatorname{supp}(\pi \sigma) \subset U_{0}$ and $\operatorname{supp}(\tau \pi \sigma) \subset U_{0}$ and because $\pi$ is a refinement map $\operatorname{supp}(\pi \sigma) \subset U_{0}$ implies $\operatorname{supp}(\sigma) \subset U_{0}$.

The last condition we have to check is (3.2.2). The chain homomorphism $c: \Delta C(\mathscr{V}) \rightarrow \Delta C(\mathscr{V})$ is defined as $\tau \pi$. According to the factorization axiom there is a $\mathscr{V} \leqq \mathscr{V}$ such that the outer edge triangle of the following diagram commutes up to homotopy. It follows that $c \pi^{\prime} \simeq \pi^{\prime \prime}$. This holds for all fine enough $\mathscr{V}^{\prime}$. Hence $H(c)$ maps the image of $H(X)$ in $H(\mathscr{V})$ identically onto itself.

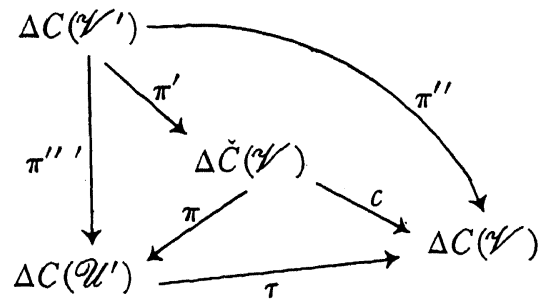

Further the various $\tau$ define (because of (3.1.3) a map of projective systems $H C\left(\mathscr{U}^{\prime}\right) \rightarrow H C(X,-)$ and hence a homomorphism $\tilde{\tau}_{*}: H C\left(\mathscr{U}^{\prime}\right) \rightarrow H(X)$. We then have $q_{\mathcal{V}} \tilde{\tau}_{*}=\tau_{*}$. Now $c_{*}=\tau_{*} \pi_{*}$ which proves that $c_{*}$ maps $H(\mathscr{V})$ into the image of $H(X)$ in $H(\mathscr{V})$. This concludes the proof of the theorem.

3.5. Remark. Theorems 3.3 and 3.4 show that the compact spaces which admit a WSC structure are precisely the compact spaces for which every finite open covering is geometric. This also shows, we feel, that the property "admits 
a WSC structure" is rather more natural than may be apparent from Thompson's original definition. Especially if we notice (cf. 4.4) that conditions (3.1.2) and (3.1.3) really say that the $H\left(\tau_{\mathscr{r}}\right)$ define a homomorphism of projective systems $H\left(\mathscr{U}^{\prime}\right) \rightarrow H C(X,-)$ such that the composed map $H C(X,-) \rightarrow$ $H\left(\mathscr{U}^{\prime}\right) \rightarrow H C(X,-)$ is the identity homomorphism (between projective systems), where the projective systems are indexed by the set of open coverings finer than $\mathscr{U}^{\prime}$.

Spaces which admit WSC structures include compact polyhedra or more generally Lefschetz's $H L C^{*}$ spaces (cf. Lefschetz [10]) and finite unions of compact convex subsets of locally convex topological vector spaces (cf. Thompson [14]).

3.6. Compactly generated Lebesgue covering dimension. Let $K$ be a compact space. We say that $K$ has Lebesgue covering dimension $\leqq n$ if for every $\mathscr{U} \in \operatorname{cov}^{f}(K)$ there is a $\mathscr{V} \in \operatorname{cov}^{f}(K), \mathscr{V} \leqq \mathscr{U}$ such that $\operatorname{dim}(C(K, \mathscr{V})) \leqq n$.

Now let $X$ be a not necessarily compact space. Then we say that $X$ has compactly generated Lebesgue covering dimension $\leqq n$ if every compact subspace $K$ of $X$ has Lebesgue covering dimension $\leqq n$. We simply write $\operatorname{dim} X$ $\leqq n$.

3.7. Proposition. If $\operatorname{dim} X \leqq n$, then every $n$-geometric cover is geometric.

Proof. Let $\mathscr{C}$ be an $n$-geometric cover and $\mathscr{U}^{\prime} \in \mathfrak{A}(\mathscr{C})$. (Note that $\operatorname{dim} C\left(X, \mathscr{U}^{\prime}\right)$ may well be larger than $n$ and it may not be possible to repair this by taking a refinement of $\mathscr{U}^{\prime}$.) Let $\Gamma, \gamma, \tau_{\mathscr{V}}$ be the corresponding structure elements:

$$
\tau_{\mathscr{r}}: C^{n+1}\left(K, \mathscr{U}^{\prime}\right) \rightarrow \Delta C(\gamma K, \mathscr{V}) .
$$

If $\mathscr{V} \in \operatorname{cov}^{f}(\gamma K)$ refines $\mathscr{U}^{\prime}$ and $\operatorname{dim} \mathscr{V} \leqq n$, then the chain map $\tau_{\mathscr{V}}$ can be extended to a chain map $\tilde{\tau}_{\mathscr{V}}: \Delta C(K, \mathscr{U}) \rightarrow \Delta C(\gamma K, \mathscr{V})$ by taking $\tilde{\tau}_{\mathscr{V}}(\sigma)=0$ if $\operatorname{dim} \sigma>n+1$. If $\mathscr{V} \in \operatorname{cov}^{f}(\gamma K)$ is any covering refining $\mathscr{U}^{\prime}$ choose a $\mathscr{V}^{\prime}$ refining $\mathscr{V}$ such that $\operatorname{dim} \mathscr{V}^{\prime} \leqq n$, and define $\tilde{\tau}_{\mathscr{V}}$ as the composite of $\bar{\tau}_{\mathscr{V}}$, and a refinement map. One easily checks that the $\tilde{\tau}_{\mathscr{V}}$ satisfy (3.1.1)-(3.1.4).

4. Consequences of the existence of geometric covers. The existence of geometric covers has strong consequences for the homology and local acyclicity of a space.

4.1. TheOREM. Let $\mathscr{C}$ be an $n$-geometric cover of the space $X$. Then the natural map $H_{k}(X) \rightarrow H_{k}\left(\mathscr{U}^{\prime}\right)$ is monomorphic for all $k \leqq n$ and $\mathscr{U}^{\prime} \in \mathfrak{U}(\mathscr{C})$. Consequently, $H_{k}(X)$ is finitely generated for all $k \leqq n$.

Proof. Let $\mathscr{U}^{\prime} \in \mathfrak{U}(\mathscr{C})$ and let $\tau, \Gamma, \gamma$ be such that the conditions (3.1.1) and (3.1.2) of 3.1 are satisfied. Let $K \in \Gamma, \mathscr{V} \leqq \mathscr{U}^{\prime}$. Then according to (3.1.2) there exists a $\mathscr{V}^{\prime} \in \operatorname{cov}^{f}(K), \mathscr{V}^{\prime} \leqq \mathscr{V}$ such that the following diagram com- 
mutes for all $k \leqq n$.

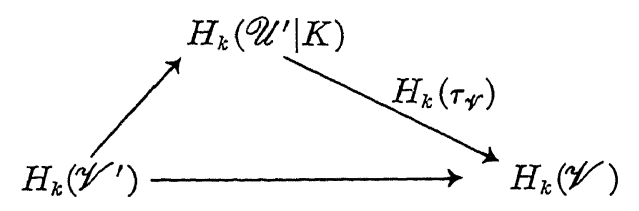

Now consider the following diagram, where the unlabelled arrows are induced by refinement maps and $i: K \rightarrow \gamma K$ is the natural inclusion.

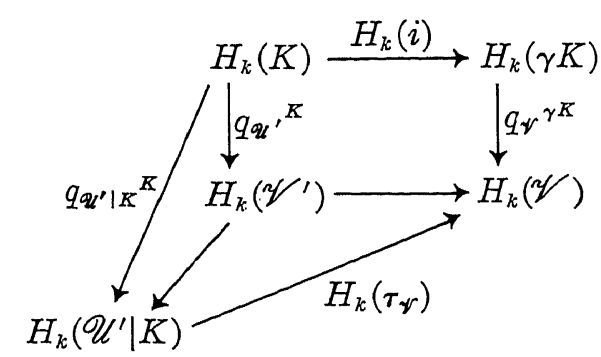

The leftmost triangle and the square are commutative by the definition of Cech homology groups, cf. 2.2 ; the lower triangle is commutative because of (3.1.2). It follows that the whole diagram is commutative.

We now have for all $z \in H_{k}(K)$

(4.1.1) $q_{\mathcal{U}^{\prime} \mid K^{K}}(z)=0 \Rightarrow H_{k}(i)(z)=0$.

Indeed if $q_{\mathscr{U} l^{\prime} \mid K^{K}}(z)=0$ then $q_{\mathfrak{r}^{\gamma}}{ }^{K} H_{k}(i)(z)=0$ for all $\mathscr{V} \in \operatorname{cov}^{f}(\gamma K)$ refining $\mathscr{U}^{\prime} \mid \gamma K$. It follows that $H_{k}(i)(z)=0$.

Now let $z \in H_{r}(X)$ and suppose that $q_{\mathscr{U}^{\prime}}(z)=0$. Since $\Gamma$ is cofinal there is a $K \in \Gamma$ such that $z$ comes from $K$, i.e. $K$ is such that $z \in \operatorname{Im}\left(H_{k}(K) \rightarrow\right.$ $\left.H_{k}(X)\right)$. Taking a larger $K \in \Gamma$ if necessary we can assume that $C\left(K, \mathscr{U}^{\prime}\right)=$ $C\left(X, \mathscr{U}^{\prime}\right)$ (i.e. if $U_{1} \cap \ldots \cap U_{r} \neq \phi, U_{i} \in \mathscr{U}^{\prime}$, then $U_{1} \cap \ldots \cap U_{r} \cap K \neq$ $\emptyset$ ). It follows (cf. 2.3) that $q_{K \mid \ell^{\prime}}{ }^{K}(\bar{z})=0$ for all $\bar{z} \in H_{k}(K)$ mapping onto $z \in H_{k}(X)$ and hence that $z=0$ because of (4.1.1).

4.2. CoRollary. If $\mathscr{C}$ is a geometric cover of a space $X$ then $H_{*}(X)$ is finitely generated. In particular $H_{k}(X)=0$ for $k$ large enough.

4.3. Remarks. The "uniformity" of $\mathscr{U}^{\prime}$ with respect to $K \in \Gamma$ and dimension $n$ is essential for these results.

Note that properties (3.1.3) and (3.1.4) of a geometric cover have not been used.

4.4. Remark. Property (3.1.3) says that the maps $H_{k}\left(\tau_{\varkappa^{*}}\right)$ define a morphism of pro-objects

$$
H_{k}\left(\mathscr{U}^{\prime} \mid K\right) \rightarrow H_{k} C(\gamma K,-)
$$

and property (3.1.2) then says that the composition

$$
H_{k} C(K,-) \rightarrow H_{k}\left(\mathscr{U}^{\prime} \mid K\right) \rightarrow H_{k} C(\gamma K,-)
$$


is the natural homomorphism of pro-objects induced by the inclusion $K \hookrightarrow \gamma K$.

It follows that the composed homomorphism

$$
H_{k}(X) \longrightarrow H_{k}\left(\mathscr{U}^{\prime}\right) \stackrel{\tau *}{\longrightarrow} H_{k}(\gamma K) \stackrel{i_{\gamma K}}{\longrightarrow} H_{k}(X)
$$

is the identity for $K$ large enough. (The last map is induced by the inclusion $\gamma K \rightarrow X$.) Indeed, because $H_{k}(X)$ is finitely generated there it a compact set $K$ such that the natural map $i_{K}: H_{k}(K) \rightarrow H_{k}(X)$ is surjective.

Let $x \in H_{k}(X), x^{\prime} \in H_{k}(K)$ such that $i_{K}\left(x^{\prime}\right)=x$. Then because $q(x)=$ $q_{\mathscr{O}^{\prime}}{ }^{K}\left(x^{\prime}\right)$ (cf. 2.3) and (4.4.1) above we have

$$
i_{\gamma K} \tau_{*} q(x)=i_{\gamma K} \tau_{*} q_{\mathscr{U}^{\prime}}{ }^{K}\left(x^{\prime}\right)=x \text {. }
$$

NB. The homomorphism $i_{\gamma K} \circ \tau_{*}$ may depend on $K$.

4.5. Proposition. Let $\mathscr{C}$ be an n-geometric cover of a space $X$ and let $\mathscr{U}^{\prime} \in$ $\mathfrak{H}(\mathscr{C})$. Then $\tilde{H}_{k}\left(U^{\prime}\right) \rightarrow \tilde{H}_{k}(X)$ is the zero map for all $U^{\prime} \in \mathscr{U}^{\prime}, k=0,1, \ldots, n$.

Proof. Let $U^{\prime} \in \mathscr{U}^{\prime}$ and let $K \subset U^{\prime}$ be compact. We have to show that there exists a $K^{\prime} \subset X$ such that the inclusion $K \rightarrow K^{\prime}$ induces the zero map on reduced homology. Take $K^{\prime}=\gamma K$. Because $K \subset U^{\prime}$ we have $\mathscr{U}^{\prime} \mid K \leqq\{K\} \leqq$ $\mathscr{U}^{\prime} \mid K$ and hence $\tilde{H}\left(\mathscr{U}^{\prime} \mid K\right)=0$. But from the proof of theorem (4.1) we have

(4.5.1) $q_{\mathscr{U}^{\prime} \mid K^{K}}(z)=0 \Rightarrow H_{k}(i)(z)=0 \quad$ for $z \in H_{k}(K)$

The same holds for reduced homology (by using the Lefschetz theorem for reduced homology). This proves the proposition.

5. Geometric covers and almost fixed points. We are now in a position to state and prove a Lefschetz type almost-fixed-point theorem.

5.1. Lefschetz number. If $X$ is a space such that $H_{*}(X)$ is finitely generated and $f: X \rightarrow X$ is continuous, we define $L(f)=\sum(-1)^{k} \operatorname{Tr}\left(H_{k}(f)\right)$ where $\operatorname{Tr}(g)$ denotes the trace of a linear map $g$ between (finite dimensional) vector spaces.

5.2. Lemma. Let $\mathscr{C}$. be a finite closed covering of a space $X$ and let $\mathscr{U}$ be a finite open covering of $X$ such that $\mathscr{C} \leqq \mathscr{U}$. For each $C \in \mathscr{C}$ choose $U_{C} \in \mathscr{U}$ such that $C \subset U_{C}$. Then there exists a finite open covering $\mathscr{U}^{\prime}$ of $X$ such that $U^{\prime} \in \mathscr{U}^{\prime}$ and $U^{\prime} \cap C \neq \emptyset$ imply $U^{\prime} \subset U_{C}$.

Proof. For each partition $\mathscr{C}=\mathscr{A} \cup \mathscr{B}$ of $\mathscr{C}$ into two disjoint parts we define the open set

$$
U_{\mathscr{A}, \mathscr{B}}^{\prime}=\left(\bigcap_{C \in \mathscr{A}} X \backslash C\right) \cap\left(\bigcap_{C \in \mathscr{B}} U_{C}\right)
$$

Take for $\mathscr{U}^{\prime}$ the covering consisting of the nonempty $U_{\mathscr{L}}^{\prime} \mathscr{B}$. $\left(\mathscr{U}^{\prime}\right.$ is a covering because $x \in U_{\mathscr{A}_{x}, \mathscr{B}_{x}}$ with $\left.\mathscr{B}_{x}=\{C \in \mathscr{C} \mid x \in C\}, \mathscr{A}_{x}=\{C \in \mathscr{C} \mid x \notin C\}\right)$.

5.3. Theorem. Let $X$ be a space with a closed geometric covering $\mathscr{C}$, and let $f: X \rightarrow X$ be continuous. Then for every finite open covering $\mathscr{U} \geqq \mathscr{C}$ we have $L(f) \neq 0 \Rightarrow$ there exists $U \in \mathscr{U}$ such that $U \cap f(U) \neq \emptyset$. 
Proof. $\mathscr{C}$ is a geometric cover. Let $\mathscr{U} \geqq \mathscr{C}$. For each $C \in \mathscr{C}$ choose $U_{C} \in \mathscr{U}$ such that $C \subset U_{C}$. Now choose $\mathscr{U}^{\prime} \in \mathfrak{A}(\mathscr{C})$ such that $U^{\prime} \in \mathscr{U}^{\prime}$ and $U^{\prime} \cap C \neq$ $\emptyset$ imply $U^{\prime} \subset U_{C}$. Such a $\mathscr{U}^{\prime}$ can be found by 5.2 , and because any refinement of a cover in $\mathfrak{I}(\mathscr{C})$ is also in $\mathfrak{A}(\mathscr{C})$ (cf. 3.1). Let $n=\operatorname{dim} C\left(X, \mathscr{U}^{\prime}\right)$. The cover $\mathscr{C}$ is $n$-geometric. Let $\Gamma, \gamma, \tau_{\mathscr{r}}$ be the other structure elements corresponding to $\mathscr{U}^{\prime}$ which go into the definition of an $n$-geometric covering.

Assume that $U \cap f(U)=\emptyset$ for all $U \in \mathscr{U}$. We are going to prove that $L(f)=0$. Let $K$ be compact such that $C\left(K, \mathscr{U}^{\prime}\right)=C\left(\mathscr{U}^{\prime}\right)$ and such that $H(K) \rightarrow H(X)$ is surjective. Let $K^{\prime} \supset \gamma K$ be such that $f(\gamma K) \subset K^{\prime}$. Let $\mathscr{V}$ be a finite open covering of $\gamma K$ such that $\mathscr{V} \leqq \mathscr{U}^{\prime}$ and such that for each $V \in \mathscr{V}$ there is a $U^{\prime} \in \mathscr{U}^{\prime}$ such that $f(V) \subset U^{\prime}$. (This can be done because $\gamma K$ is compact.) Then we have an induced chain map

$$
f .: C(\mathscr{V}) \rightarrow C\left(\mathscr{U}^{\prime}\right)=C\left(\mathscr{U}^{\prime}, K^{\prime}\right)
$$

and composing this with $\tau_{\mathscr{V}}: C\left(\mathscr{U}^{\prime}\right) \rightarrow \Delta C(\mathscr{V})$ we obtain an induced chain map

$$
f_{. .}: \Delta C\left(\mathscr{U}^{\prime}\right) \rightarrow \Delta C\left(\mathscr{U}^{\prime}\right)
$$

On the other hand we have a map of pro-objects (cf. 4.4) $\tau_{* k}{ }^{K}: H_{k}\left(\mathscr{U}^{\prime} \mid K\right)$ $\rightarrow H_{k}(\gamma K,-)$ and a homomorphism induced by $f, f_{* k} \gamma^{K}: H_{k}(\gamma K,-) \rightarrow$ $H_{k}\left(K^{\prime},-\right)$. Composing this and taking the projective limit gives a homomorphism

$$
f_{* \gamma^{\gamma}}{ }^{K} \circ \tau_{* k}{ }^{K}: H_{k}\left(\mathscr{U}^{\prime}\right)=H_{k}\left(\mathscr{U}^{\prime} \mid K\right) \rightarrow H_{k}\left(K^{\prime}\right)
$$

Composing this with the natural map $H_{k}\left(K^{\prime}\right) \rightarrow H_{k}(X)$ gives us a map

$$
g_{* k}: H_{k}\left(\mathscr{U}^{\prime}\right) \rightarrow H_{k}(X)
$$

Now consider the following diagram

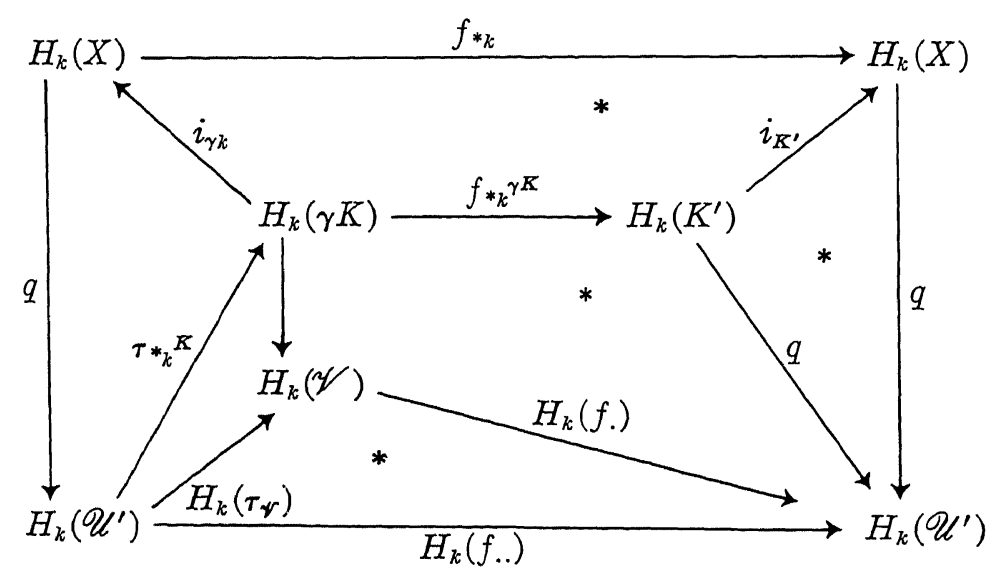

The starred triangles and quadrangles are commutative and by (4.4) we have that $i_{\gamma k} \tau_{* k}{ }^{K} q=$ id (left-most triangle). Retaining only what we need, we find 
a diagram

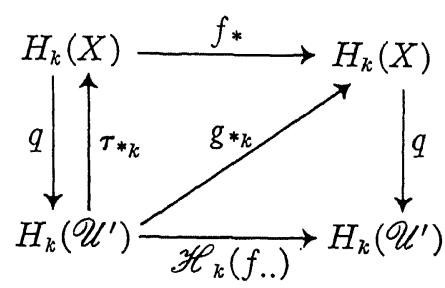

We have $q g_{* k}=H_{k}(f .$.$) and f_{*} \tau_{* k}=g_{* k}$. Hence $g_{* k} q=f_{*} \tau_{* k} q=f_{*}$ and $q f_{*}=H_{k}(f .)$.$q .$

It follows that $H_{k}(f .).\left(H_{k}\left(\mathscr{U}^{\prime}\right)\right) \subset H_{k}(X) \subset H_{k}\left(\mathscr{U}^{\prime}\right)$, where $H_{k}(X)$ is seen as a subvectorspace of $H_{k}\left(\mathscr{U}^{\prime}\right)$ by means of the injection $q_{\mathscr{U}^{\prime}}$ (with inverse projection $\tau_{* k}$, cf 4.4). And from this it follows that

(5.3.2) $\operatorname{Tr}\left(H_{k}(f .).\right)=\operatorname{Tr}\left(f_{*_{k}}\right)$ for all $k$.

Now by (3.1.4) there is for every $\sigma \in C\left(\mathscr{U}^{\prime} \mid K\right)$ an element $C \in \mathscr{C}$ such that $\operatorname{rsupp}(\sigma) \subset C$ and $\operatorname{rsupp}\left(\sigma^{\prime}\right) \cap C \neq \emptyset$ for all $\sigma^{\prime}$ in $\tau_{\mathscr{x}}(\sigma)$.

It follows that (because $U^{\prime} \in \mathscr{U}^{\prime}$ and $U^{\prime} \cap C \neq \emptyset$ imply $U^{\prime} \subset U_{C}$ )

$\operatorname{supp}(\sigma) \subset U_{C}$ and $\operatorname{supp}\left(\sigma^{\prime}\right) \subset U_{C}$ for all $\sigma^{\prime}$ in $\tau_{\mathscr{V}}(\sigma)$.

But $U_{C} \cap f\left(U_{C}\right)=\phi$. Hence $\sigma$ does not occur with nonzero coefficient in $f_{.} \tau_{\mathscr{V}}(\sigma)=f_{. .}(\sigma)$. Hence $\operatorname{Tr}\left(f_{. .}\right)_{k}=0$.

By the Hopf theorem and (5.3.2) we then have

$$
\begin{aligned}
& L(f)=\sum(-1)^{k} \operatorname{Tr}\left(f_{*_{k}}\right)=\sum(-1)^{k} \operatorname{Tr}\left(K_{k}(f . .)\right) \\
& \quad=\sum(-1)^{k} \operatorname{Tr}\left(\left(f_{. .}\right)_{k}\right)=0 .
\end{aligned}
$$

5.4. Remark. This proof is quite similar in spirit to the proofs of various other Lefschetz type fixed point theorems (cf. eg. Thompson [12]).

5.5. Addendum. It is possible to extend Theorem (5.2). A closed continuous surfective map $f: Y \rightarrow X$ is called a Vietoris map if the subspace $f^{-1}(x)$ is homologically trivial (with respect to $H$ ) for all $x \in X$. The extended version of Theorem (5.2) then reads

Theorem. Let $X$ be a normal space and $\mathscr{C}$ a closed geometric cover of $X$. Let $Y$ be a topological space and $f, g: Y \rightarrow X$ two continuous maps of which $f$ is a Vietoris map. Then if $L(f, g) \neq 0$ then for every finite open cover $\mathscr{U} \geqq \mathscr{C}$ there is an $y \in Y$ and $U \in \mathscr{U}$ such that $f(y) \in U, g(y) \in U$.

Here $L(f, g)$ is defined as $L(f, g)=\sum(-1)^{k} \operatorname{Tr}\left(H_{k}(g) H_{k}(f)^{-1}\right)$ which makes sense because the Vietoris map $f$ induces isomorphisms on the homology groups.

This theorem allows one to deal with multifunctions $F: X \rightarrow X$ and gives as a corollary an Eilenberg-Montgomery type (cf. [5]) fixed point theorem by taking $X$ compact (cf. the introduction). 
The chief technical difficulty in proving this theorem (as compared to Theorem 5.2) lies in the obtaining of $H_{k}(f)^{-1}$ as $H_{k}$ of a suitably controlled chain map. For details, cf. [15].

6. Local connectedness. As in the case of e.g. WSC structures (cf. Thompson $[\mathbf{1 2}]$ and $[\mathbf{1 3}]$ ), some kind of local $n$-connectedness (with respect to $H$ ) is related to the existence of $n$-geometric covers. This and the following sections are concerned with this connection.

6.1. (Partial) Realizations. Let $S_{1} \subset S$ be a pair of simplicial complexes; $S_{1}$ is said to be dense in $S$ if $S_{1}{ }^{0}$, the zero skeleton of $S_{1}$, is equal to $S^{0}$.

Let $\mathscr{W}$ be a finite (open) cover of a space $X$. A partial realization of $S$ in $\mathscr{W}$ is an augmentation preserving chain map

$$
\tau_{1}: S_{1} \rightarrow \Delta C(X, \mathscr{W})
$$

where $S_{1}$ is dense subcomplex of $S$. If $S_{1}=S$ we speak of a (full) realization.

If $\sigma$ is a simplex of $S$, then $S(\sigma)$ denotes the subcomplex of $S$ consisting of all faces of $\sigma$ (including $\sigma$ itself).

The partial realization $\tau_{1}: S_{1} \rightarrow \Delta C(X, \mathscr{W})$ is said to be of norm $\leqq \mathscr{C}$, where $\mathscr{C}$ is another covering of $X$, if for every $\sigma \in S$ there is a $C \in \mathscr{C}$ such that $\tau_{1}(\mu)$ is on $C$ for all $\mu \in S_{1} \cap S(\sigma)$.

Let $X_{2} \subset X_{1}$ be a pair of topological spaces; let $\mathscr{C}_{1}, \mathscr{U}_{1}$ and $\mathscr{C}_{2}, \mathscr{U}_{2}$ be finite covers of $X_{1}, X_{2}$ respectively with $\mathscr{U}_{1}$ and $\mathscr{U}_{2}$ open covers and $\mathscr{C}_{2} \leqq \mathscr{C}_{1}$, $\mathscr{U}_{2} \leqq \mathscr{U}_{1}$. Then we say that the pair $\left(\mathscr{U}_{1}, \mathscr{U}_{2}\right)$ has enough controlled realizations for dimensions $\leqq n\left(\left(\mathscr{U}_{1}, \mathscr{U}_{2}\right)\right.$ has $\left.\operatorname{ECR}(n)\right)$ with respect to $\left(\mathscr{C}_{1}, \mathscr{C}_{2}\right)$ if, for every $S_{1} \subset S$, every partial realization $\tau_{1}: S_{1} \rightarrow \Delta C\left(X_{2}, \mathscr{U}_{2}\right)$ of norm $\leqq \mathscr{C}_{2}$ extends to a partial realization $\tau: S^{n} \cup S_{1} \rightarrow \Delta C\left(X_{1}, \mathscr{U}_{1}\right)$ of norm $\leqq \mathscr{C}_{1}$. That is, we have a commutative diagram

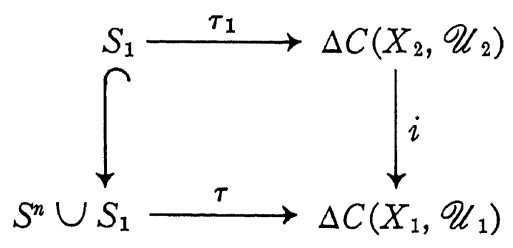

for some suitable refining homomorphism $i$.

6.2. $l c^{n}$ and $c-l c^{n}$ refinements. Let $X_{2} \subset X_{1}$ be a pair of topological spaces and let $\mathscr{C}_{2}, \mathscr{C}_{1}$ be covers of $X_{2}, X_{1}$ respectively such that $\mathscr{C}_{2} \leqq \mathscr{C}_{1}$. Then $\mathscr{C}_{2}$ is an $l c^{n}$ refinement of $\mathscr{C}_{1}$ if for every $\mathscr{U}_{1} \in \operatorname{cov}^{f}\left(X_{1}\right)$ there exists a $U_{2} \in$ $\operatorname{cov}^{f}\left(X_{2}\right)$ such that $\mathscr{U}_{2} \leqq \mathscr{U}_{1}$ and $\left(\mathscr{U}_{1}, \mathscr{U}_{2}\right)$ has $E C R(n+1)$ with respect to $\left(\mathscr{C}_{1}, \mathscr{C}_{2}\right)$. If $\mathscr{C}_{2}$ is an $l c^{n}$ refinement of $\mathscr{C}_{1}$ for every $n, \mathscr{C}_{2}$ is said to be an lc refinement of $\mathscr{C}_{1}$ (NB. $l c^{n}$ corresponds to $E C R(n+1)$ ).

Let $X$ be a space and let $\mathscr{C}_{2} \leqq \mathscr{C}_{1}$ be covers of $X$. We say that $\mathscr{C}_{2}$ is a $c-l c^{n}$ refinement of $\mathscr{C}_{1}$ if for every compact set $K_{2} \subset X$ there is a larger 
compact set $K_{1}$ in $X$ such that $\mathscr{C}_{2} \mid K_{2}$ is an $l c^{n}$ refinement of $\mathscr{C}_{1} \mid K_{1}$. If $\mathscr{C}_{2}$ is an $c-l c^{n}$ refinement of $\mathscr{C}_{1}$ for every $n, \mathscr{C}_{2}$ is said to be an $c-l c^{\omega}$ refinement of $\mathscr{C}_{1}$.

6.3. Elementary properties. Let $X_{3} \subset X_{2} \subset X_{1}$ and let $\mathscr{C}_{3}, \mathscr{C}_{2}, \mathscr{C}_{1}$ be covers of $X_{3}, X_{2}, X_{1}$ respectively such that $\mathscr{C}_{3} \leqq \mathscr{C}_{2} \leqq \mathscr{C}_{1}$. Then

(i) If $\mathscr{C}_{2}$ is an $l c^{n}\left(l c^{\omega}\right)$ refinement of $\mathscr{C}_{1}$ then so is $\mathscr{C}_{3}$. of $\mathscr{C}_{1}$

(ii) If $\mathscr{C}_{3}$ is an $l c^{r}\left(l c^{\omega}\right)$ refinement of $\mathscr{C}_{2}$ then it is also an $l c^{n}\left(l c^{\omega}\right)$ refinement

Let $\mathscr{C}_{3} \leqq \mathscr{C}_{1}$ be two covers of a compact space $X$. Then

(iii) $\mathscr{C}_{2}$ is an $c-l c^{n}\left(\right.$ resp. $\left.c-l c^{\omega}\right)$ refinement of $\mathscr{C}_{1}$ if and only if $\mathscr{C}_{2}$ is an $l c^{n}\left(\right.$ resp. $\left.l c^{\omega}\right)$ refinement of $\mathscr{C}_{1}$.

6.4. $l c^{n}$ spaces and $l c^{n}$ and $c-l c^{n}$ covers. A compact space is said to be $l c^{n}$ (resp. $l c^{\omega}$ ) if every finite open cover has an $l c^{n}$ (resp. $l c^{\omega}$ ) refinement.

A covering $\mathscr{C}$ of a space $X$ is $c-l c^{n}$ (resp. $\left.l c^{n}\right)$ if it is a $c-l c^{n}$ (resp. $\left.l c^{n}\right)$ refinement of the trivial cover.

One could perfectly well define what a $c-l c^{n}\left(\right.$ resp. $\left.c-l c^{\omega}\right)$ space would be. But there seem to be very few examples which are noncompact; we know none. The property $l c^{\omega}$ seems somewhat weaker than $l c^{*}$ (cf. e.g. Begle [1] or Thompson [12] for a definition of $l c^{*}$ ).

7. Acyclicity and $c-l c^{n}$ refinements. We have seen (cf. 4.5) that some kind of local acyclicity is implied by the existence of $n$-geometric covers. On the other hand given acyclicity properties of a suitable kind one can go a fair way towards the construction of $n$-geometric covers as we shall attempt to show in this and the next section. The first step is to show that given suitable acyclicity conditions, partial realizations can be extended. One has even better control over the supports than is needed for $c-l c^{n}$ refinements and this results in some "uniqueness up to homotopy" statements and these in turn will permit us to construct $n$-geometric covers in Section 8 .

7.1. Lemma. Let $K_{2} \subset K_{1}$ be compact spaces and suppose that for a certain $n$ the induced homomorphism $\widetilde{H}_{n}\left(K_{2}\right) \rightarrow \widetilde{H}_{n}\left(K_{1}\right)$ is trivial. Then for every finite open cover $\mathscr{U}_{1}$ of $K_{1}$ there is a finite open cover $\mathscr{U}_{2}$ of $K_{2}$ such that $\mathscr{U}_{2} \leqq \mathscr{U}_{1}$ and

$$
\widetilde{H}_{n}\left(K_{2}, \mathscr{U}_{2}\right) \rightarrow \widetilde{H}_{n}\left(K_{1}, \mathscr{U}_{1}\right)
$$

is the trivial map.

Proof. We have a commutative diagram

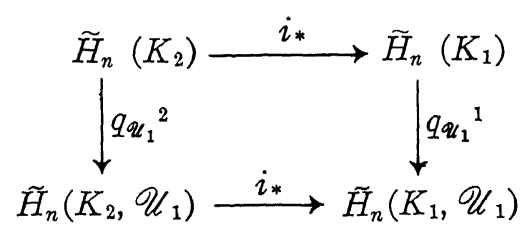


where $i: K_{2} \rightarrow K_{1}$ is the inclusion. By the Lefschetz theorem (cf. (2.5)) there is a finite open cover $\mathscr{U}_{2}$ or $K_{2}$ refining $\mathscr{U}_{1}$ such that

$$
\operatorname{Im}\left(\tilde{H}_{n}\left(K_{1}, \mathscr{U}_{2}\right) \rightarrow \widetilde{H}_{n}\left(K_{2}, \mathscr{U}_{1}\right)\right)=\operatorname{Im}\left(\widetilde{H}_{n}\left(K_{2}\right) \rightarrow \widetilde{H}_{n}\left(K_{2}, \mathscr{U}_{1}\right)\right) .
$$

It follows that the natural map $\widetilde{H}_{n}\left(K_{2}, \mathscr{U}_{2}\right) \rightarrow \widetilde{H}_{n}\left(K_{1}, \mathscr{U}_{1}\right)$ is trivial. Note that any refinement $\mathscr{U}_{2}^{\prime}$ of $\mathscr{U}_{2}$ also works.

7.2. Definition and construction. Let $X_{2} \subset X_{1}$ be topological spaces and let $\mathscr{C}_{2}, \mathscr{C}_{1}$ be finite covers of $X_{2}$ and $X_{1}$ respectively such that $\mathscr{C}_{2} \leqq \mathscr{C}_{1}$. Let $j: \mathscr{C}_{2} \rightarrow \mathscr{C}_{1}$ be a refinement map. We say that $j$ is acyclic in dimension $n$ if for all subsets $\mathscr{A}_{2} \subset \mathscr{C}_{2}$ and all compact subsets $K_{2} \subset \cap \mathscr{A}_{2}$ there is a compact subset $K_{1} \subset \cap j\left(\mathscr{A}_{2}\right)$ such that $K_{2} \subset K_{1}$ and such that

$$
\tilde{H}_{n}\left(K_{2}\right) \rightarrow \tilde{H}_{n}\left(K_{1}\right) \text { is the zero map, }
$$

Now let $n_{0}$ and $n$ be nonnegative integers. Suppose we have a sequence of finite closed covers $\mathscr{C}_{0} \leqq \mathscr{C}_{1} \ldots \leqq \mathscr{C}_{n+1}$ of a space $X$ with refinement maps $j_{k}$ : $\mathscr{C}_{k} \rightarrow \mathscr{C}_{k+1}, k=0,1, \ldots, n$ such that $j_{k}$ is acyclic in dimension $n_{0}+k$.

Let $K$ be any compact subset of $X$. Then there exists a sequence of compact subsets

(7.2.2) $\quad K=K_{0} \subset K_{1} \subset \ldots \subset K_{n+1}$

such that for all $\mathscr{A}_{k} \subset \mathscr{C}_{k}$ such that $K_{k} \cap\left(\cap \mathscr{A}_{k}\right) \neq \emptyset$,

(7.2.3) $\quad \widetilde{H}_{n_{0}+k}\left(K_{k} \cap\left(\cap \mathscr{A}_{k}\right)\right) \rightarrow \widetilde{H}_{n_{0}+k}\left(K_{k+1} \cap\left(\cap j_{k}\left(\mathscr{A}_{k}\right)\right)\right)$ is the zero map and consequently for every finite open cover $\mathscr{V}_{n+1}$ of $K_{n+1}$ there exists a sequence of open covers $\mathscr{V}_{k}, k=0,1, \ldots, n$,

$$
\mathscr{V}_{0} \leqq \mathscr{V}_{1} \leqq \mathscr{V}_{2} \leqq \ldots \leqq \mathscr{V}_{n+1}
$$

such that for all $\mathscr{A}_{k} \subset \mathscr{C}_{k}$ such that $K_{k} \cap\left(\cap \mathscr{A}_{k}\right) \neq \emptyset$,

$$
\widetilde{H}_{n_{0}+k}\left(K_{k} \cap\left(\cap \mathscr{A}_{k}\right), \mathscr{V}_{k}\right) \rightarrow \widetilde{H}_{n_{0}+k}\left(K_{k+1} \cap\left(\cap j_{k} \mathscr{A}_{k}\right), \mathscr{V}_{k+1}\right)
$$

is the zero map.

The sequence $(7.2 .2)$ is constructed as follows. Suppose we have found $K_{k}$, $k \geqq 0$. For every $\mathscr{A}_{k} \subset \mathscr{C}_{k}$ such that $K_{k} \cap\left(\cap \mathscr{A}_{k}\right) \neq \emptyset$ let $\left.K_{k}{ }^{\prime} \mathscr{A}_{k}\right)$ be a compact set containing $K_{k} \cap\left(\cap \mathscr{A}_{k}\right)$ and contained in $\cap j_{k}\left(\mathscr{A}_{k}\right)$ such that (7.2.1) is satisfied.

Now let $K_{k+1}$ be the union of all the $K_{k}{ }^{\prime}\left(\mathscr{A}_{k}\right)$. Then of course $K_{k+1} \cap$ $\left(\cap j_{k} \mathscr{A}_{k}\right) \supset K_{k}{ }^{\prime}\left(\mathscr{A}_{k}\right)$ so that (7.2.3) is satisfied. To find the sequence (7.2.4) such that (7.2.5) is satisfied, apply 7.1 repeatedly.

7.3. Proposition. Le $\mathscr{C}_{0} \leqq \ldots \leqq \mathscr{C}_{n+1}$ be a sequence of finite closed covers of a space $X$ with corresponding refinement maps $j_{k}: \mathscr{C}_{k} \rightarrow \mathscr{C}_{k+1}$ such that $j_{k}$ is acyclic in dimension $n_{0}+k$. Let $\mathscr{C}_{0}^{\prime} \leqq \mathscr{C}_{0}$ have a refinement map $i: \mathscr{C}_{0}^{\prime} \rightarrow \mathscr{C}_{0}$ such that $C_{0}{ }^{\prime} \subset$ interior $\left(i\left(C_{0}{ }^{\prime}\right)\right)$ for all $C_{0}{ }^{\prime} \in \mathscr{C}_{0}{ }^{\prime}$. Let $S_{1} \subset S$ be a pair of (finite) simplicial complexts. 
Let $K=K_{0} \subset K_{1} \subset \ldots \subset K_{n+1}$ be as in 7.2. Then for every $\mathscr{V}_{n+1} \in$ $\operatorname{cov}^{f}\left(K_{n+1}\right)$ there exists a sequence $\mathscr{V}_{0} \leqq \mathscr{V}_{1} \leqq \ldots \leqq \mathscr{V}_{n+1}, \mathscr{V}_{k} \in \operatorname{cov}^{f}\left(K_{k}\right)$ such that for every partial realization

$$
\tau_{0}: S^{n_{0}} \cup S_{1} \rightarrow \Delta C\left(K_{0}, \mathscr{V}_{0}\right)
$$

of norm $\leqq \mathscr{C}_{0}{ }^{\prime}$ there exists a sequence of partial realizations

$$
\tau_{k}: S^{n_{0}+k} \cup S_{1} \rightarrow \Delta C\left(K_{k}, \mathscr{V}_{k}\right), \quad k=0, \ldots, n+1
$$

such that

$$
\begin{aligned}
& \tau_{k+1} \text { extends } \tau_{k}, \quad k=0,1, \ldots, n \\
& \text { if } \sigma \text { is a simplex of } S^{n_{0}+k} \cup S_{1} \text { then } \tau_{k}(\sigma) \text { is on } \cap \mathscr{A}_{k}(\sigma) \cap K_{k}, \\
& k=0,1, \ldots, n+1,
\end{aligned}
$$

where $\mathscr{A}_{k}(\sigma)=j_{k-1} \ldots j_{0} i \mathscr{A}(\sigma)$, and $\mathscr{A}(\sigma)$ is defined as follows. Let $\sigma_{1}, \ldots, \sigma_{s}$ be the maximal simplices of $S$. For each $\sigma_{r}$ choose a $C_{0}{ }^{\prime}(r)$ such that $\tau_{0}\left(\left(S^{n_{0}} \cup S_{1}\right) \cap S\left(\sigma_{r}\right)\right)$ is on $C_{0}{ }^{\prime}(r)$. (Such $C_{0}{ }^{\prime}(r)$ exist because $\tau_{0}$ is of norm $\leqq$ $\mathscr{C}_{0}{ }^{\prime}$.) Now define $\mathscr{A}(\sigma)=\left\{C_{0}{ }^{\prime}(r) \mid \sigma\right.$ is a face of $\left.\sigma_{r}\right\}$.

Moreover in the case $n_{0}=0$ there is the following homotopy property: If $\mathscr{V}_{0} \leqq \mathscr{V}_{1}^{\prime} \leqq \ldots \leqq \mathscr{V}_{n}^{\prime} \leqq \mathscr{V}_{n+1}$ is a second series of refinements and $\tau_{0}{ }^{\prime}$, $\tau_{1}{ }^{\prime}, \ldots, \tau_{n+1}{ }^{\prime}$ a second series of extensions such that (7.3.1) and (7.3.2) hold and if $\tau_{0}^{\prime}=\tau_{0}$ on $S_{1}$ then $H_{k}\left(\tau_{n+1}^{\prime}\right)=H_{k}\left(\tau_{n+1}\right)$ for $k=0,1, \ldots, n$.

Remarks. 1. If $n_{0}>0$ and $\tau_{0}=\tau_{0}^{\prime}$ then also $H_{k}\left(\tau_{n+1}^{\prime}\right)=H_{k}\left(\tau_{n+1}\right)$ for $k=$ $0, \ldots, n_{0}+n$.

2. In general there are several different choices for the $C_{0}{ }^{\prime}(r)$ and correspondingly one finds different $\mathscr{A}(\sigma)$ and different $\tau_{k}$.

The proof of Proposition 7.3 is in several steps: subsections 7.4-7.7. The first step is to choose $\mathscr{V}_{0} \leqq \ldots \leqq \mathscr{V}_{n+1}$ such that (7.3.2) holds for $k=0$.

7.4. Remarks on the $\mathscr{A}(\sigma)$ and the sequence $\mathscr{V}_{0} \leqq \ldots \leqq \mathscr{V}_{n+1}$. Choose a sequence of covers $\mathscr{V}_{0} \leqq \mathscr{V}_{1} \leqq \ldots \leqq \mathscr{V}_{n+1}, \mathscr{V}_{k} \in \operatorname{cov}^{f}\left(K_{k}\right)$ such that $(7.2 .5)$ is satisfied. Refining $\mathscr{V}_{0}$ if necessary we can also assume that $V_{0} \in \mathscr{V}_{0}$, $V_{0} \cap C_{0}{ }^{\prime} \neq \emptyset \Rightarrow V_{0} \subset i\left(C_{0}{ }^{\prime}\right) \in \mathscr{C}_{0}$.

Let $\sigma$ be a simplex of $S^{n_{0}} \cup S_{1}$. Let $\sigma$ be a face of $\sigma_{r}$. Then $\tau_{0}(\mu)$ is on $C_{0}{ }^{\prime}(r)$ for all vertices $\mu$ of $\sigma$. Hence $V_{0} \cap C_{0}{ }^{\prime}(r) \neq \emptyset$ for all $V_{0}$ occurring in $\tau_{0}(\mu)$, hence $V_{0} \subset i\left(C_{0}{ }^{\prime}(r)\right)$ for all vertices $V_{0}$ ocurring in $\tau_{0}(\mu), \mu$ a vertex of $\sigma$. Hence $\operatorname{supp}\left(\tau_{0}(\sigma)\right) \subset i\left(C_{0}{ }^{\prime}(r)\right)$ for all $r$ such that $\sigma \subset \sigma_{r}$ hence $\operatorname{supp}\left(\tau_{0}(\sigma)\right) \subset$ $\left(\cap \mathscr{A}_{0}(\sigma)\right) \cap K_{0}$ which certainly implies (7.3.2) which says that $\operatorname{rsupp}\left(\sigma^{\prime}\right) \cap$ $\left(\cap \mathscr{A}_{0}(\sigma)\right) \cap K_{0} \neq \emptyset$ for every simplex $\sigma^{\prime}$ occurring in $\tau_{0}(\sigma)$.

Note that

(7.4.1) $\mu$ a face of $\sigma \Rightarrow \mathscr{A}(\mu) \supset \mathscr{A}(\sigma)$

(for if $\sigma$ is a face of $\sigma_{r}$ then so is $\mu$ ).

7.5. Existence of the sequence of extensions $\tau_{0}, \tau_{1}, \ldots, \tau_{n+1}$. Let $\mathscr{V}_{0} \leqq \ldots \leqq$ $\mathscr{V}_{n+1}$ be the sequence of refinements of 7.4 above. We have just seen that 
$\tau_{0}$ satisfies 7.3.2. By induction we can suppose that $\tau_{k}$ has been constructed such that (7.3.1) and (7.3.2) hold.

Consider the following diagram

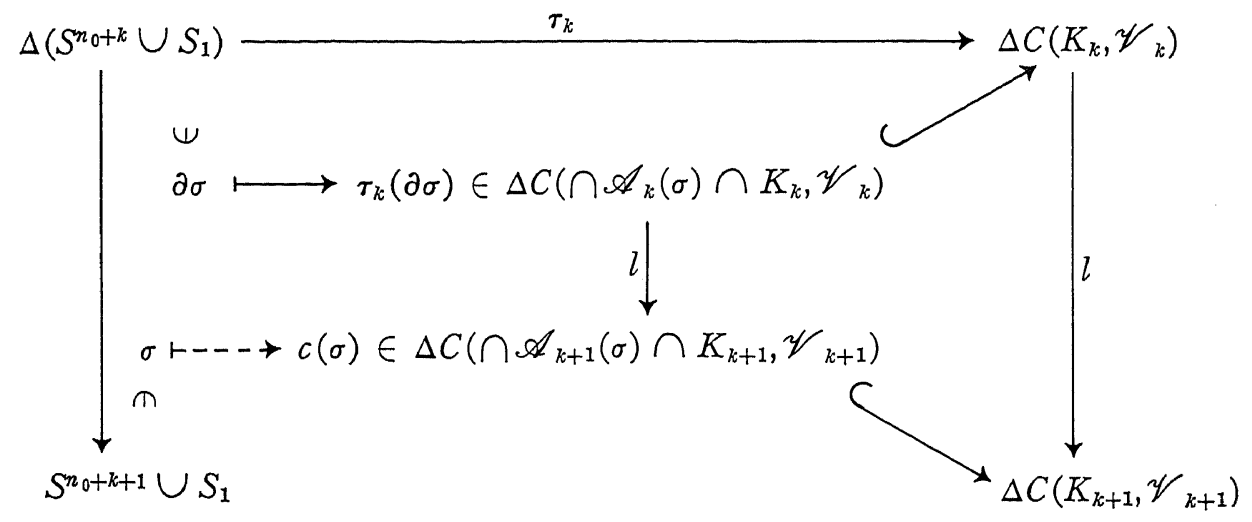

Let $\sigma \in S^{n_{0+k+1} \backslash} \backslash S^{n_{0}+k} \cup S_{1}$. Let $\mu$ be an $n_{0}+k$ face of $\sigma$. Then $\tau_{k}(\mu)$ is on $\cap \mathscr{A}_{k}(\mu) \cap K_{k}$ hence it is certainly on $\cap \mathscr{A}_{k}(\sigma) \cap K_{k}$ by (7.4.1). Therefore $\tau_{k}(\partial \sigma)$ is on $\cap \mathscr{A}_{k}(\sigma) \cap K_{k}$. The image of $\tau_{k}(\partial \sigma)$ under $l$ is homologous to zero because (7.2.5) holds (NB. $\tau_{k}(\partial \sigma)$ is a cycle because $\tau_{k}$ is an augmentation preserving chain map.) Therefore there exists a $c(\sigma)$ such that $\partial c(\sigma)=$ $l \tau_{k}(\partial \sigma)$. Now define $\tau_{k+1}(\sigma)=c(\sigma)$. Do this for every $\sigma \in S^{n_{0+k+1}} \backslash S^{n_{0+k}} \cup S_{1}$, and define $\tau_{k+1}(\mu)=l \tau_{k}(\mu)$ for $\mu \in S^{n_{0+k}} \cup S_{1}$. Note that $\tau_{k+1}$ satisfies (7.3.1) and (7.3.2).

7.6. The homotopy property for equal refining sequences. Consider the simplicial complex $S \times I$ and let $S_{2}$ be the subcomplex $S_{2}=S_{1} \times I$. Now define

(7.6.1) $T_{0}:(S \times I)^{0} \cup S_{2} \rightarrow \Delta C\left(K_{0}, \mathscr{V}_{0}\right)$

as follows. Let $e_{1}, \ldots, e_{s}$ be an ordering of the vertices of $S$. Then the simplices of $S \times I$ are all sets of the form $\left\{e_{i(1)}{ }^{0}, \ldots, e_{i(r)}{ }^{0}, e_{i(r+1)}{ }^{1}, \ldots, e_{i(t)}{ }^{1}\right\}$ such that $i(1)<\ldots<i(r) \leqq i(r+1)<\ldots<i(t)$ and $\left\{e_{i(1)}, \ldots, \epsilon_{i(t)}\right\}$ is a simplex of $S$. We now define $T_{0}$ on the vertices of $S \times I$ by $T_{0}\left(e_{i}{ }^{0}\right)=\tau_{0}\left(e_{i}\right), T_{0}\left(e_{i}{ }^{1}\right)=$ $\tau_{0}{ }^{\prime}\left(e_{i}\right)$ and on $S_{1} \times I=S_{2}$ we define $T_{0}$ by

$$
\begin{aligned}
\left.T_{0}\left(\left\{e_{i(1)}{ }^{0}, \ldots, e_{i(r)}\right)^{0}, e_{i(r+1)^{1}}, \ldots, e_{i(t)}{ }^{1}\right\}\right)= & \tau_{0}\left\{e_{i(1)}, \ldots, e_{i(t)}\right\} \\
& =\tau_{0}{ }^{\prime}\left\{e t_{i(1)}, \ldots, e_{i(t)}\right\} .
\end{aligned}
$$

Then $T_{0}$ satisfies (7.3.2). Now extend $T_{0}$ to $T_{n+1}$ exactly as we extended $\tau_{0}$ to $\tau_{n+1}$ in 7.5, taking care to define $T_{k}(\sigma)=\tau_{k}(\sigma)$ if $\sigma$ is a $k$-simplex of the form $\left\{e_{i(1)}{ }^{0}, \ldots, e_{i(k+1)}{ }^{0}\right\}$ and $T_{k}(\sigma)=\tau_{k}{ }^{\prime}(\sigma)$ if $\sigma$ is a $k$-simplex of the form $\left\{e_{i(1)^{1}}, \ldots, e_{i(k+1)}{ }^{1}\right\}$. We then have a chain map

$$
T_{n+1}:(S+I)^{n+1} \cup S_{2} \rightarrow \Delta C\left(K_{n+1}, \mathscr{V}_{n+1}\right)
$$

which restricts to $\tau_{n+1}$ on $S^{n+1} \times\{0\} \cup S_{1} \times\{0\}$ and to $\tau_{n+1}{ }^{\prime}$ on $S^{n+1} \times$ $\{1\} \cup S_{1} \times\{1\}$. This proves that $H_{k}\left(\tau_{n+1}{ }^{\prime}\right)=H_{k}\left(\tau_{n+1}\right)$ for $k=0,1, \ldots, n$. 
7.7. The homotopy property for different refining sequences. Now let $\mathscr{V}_{0} \leqq$ $\mathscr{V}_{1} \leqq \ldots \leqq \mathscr{V}_{n+1}, \mathscr{V}_{0} \leqq \mathscr{V}_{1}^{\prime} \leqq \ldots \leqq \mathscr{V}_{n}^{\prime} \leqq \mathscr{V}_{n+1}$ and $\tau_{0}, \tau_{1}, \ldots, \tau_{n+1}$; $\tau_{0}{ }^{\prime}, \tau_{1}{ }^{\prime}, \ldots, \tau_{n+1}{ }^{\prime}$ be two different sequences of refinements with corresponding $\tau_{k}$ and $\tau_{k}{ }^{\prime}$ such that (7.3.1) and (7.3.2) are satisfied. It suffices to prove that $H_{m}\left(\tau_{n+1}\right)=H_{m}\left(\tau_{n+1}{ }^{\prime}\right), m=0, \ldots, n$ in case $\mathscr{V}_{k} \leqq \mathscr{V}_{k}$ for all $k=$ $0, \ldots, n+1$. (Take a common refinement of the two refinement sequences such that (7.2.5) holds for this common refinement sequence.) Define $\tau_{k}{ }^{\prime \prime}=$ $l_{k} \circ \tau_{k}{ }^{\prime}$ where $l_{k}$ is induced by a refinement map; we can take $l_{0}=l_{n+1}=$ identity. Then $\tau_{0}, \ldots, \tau_{n+1} ; \tau_{0}{ }^{\prime \prime}, \ldots, \tau_{n+1}{ }^{\prime \prime}$ are two sequences of extensions corresponding to the same refinement sequence satisfying (7.3.1) and (7.3.2). Therefore $H_{m}\left(\tau_{n+1}\right)=H_{m}\left(\tau_{n+1}{ }^{\prime}\right)$ for $m=0,1, \ldots, n$.

7.8. Corollary. Let $\mathscr{C}_{0}^{\prime} \leqq \mathscr{C}_{0} \leqq \ldots \leqq \mathscr{C}_{n+1}$ be a sequence of closed covers with a refining map $\mathscr{C}_{k} \rightarrow \mathscr{C}_{k+1}$ which is acyclic in dimension $n_{0}+k, k \in$ $\{0, \ldots, n\}$, and such that each member of $\mathscr{C}_{0}{ }^{\prime}$ is contained in the interior of some member of $\mathscr{C}_{0}$. Let $\mathscr{C}_{0}^{\prime \prime} \leqq \mathscr{C}_{0}^{\prime}$ be a $c-l c^{n-1}$ refinement. Then $\mathscr{C}_{0}^{\prime \prime} \leqq \mathscr{C}_{n+1}$ is a $c-l c^{n_{0}+n}$ refinement. If, in particular, $n_{0}=0$, then $\mathscr{C}_{0}^{\prime} \leqq \mathscr{C}_{n+1}$ is a $c-l c^{n}$ refinement.

Proof. Let $K$ be compact, choose $K_{0}$ such that for every $\mathscr{V}_{0} \in \operatorname{cov}^{f}\left(K_{0}\right)$ there is a $\mathscr{V}_{0}^{\prime} \in \operatorname{cov}^{\prime}(K)$ such that $\left(\mathscr{V}_{0}, \mathscr{V}_{0}^{\prime}\right)$ has $\operatorname{ECR}\left(n_{0}\right)$ with respect to $\left(\mathscr{C}_{0}^{\prime}, \mathscr{C}_{0}^{\prime \prime}\right)$. Now let $K_{0} \subset K_{1} \subset \ldots \subset K_{n+1}$ be as in 7.2 . For every $\mathscr{V}_{n+1} \in$ $\operatorname{cov}^{f}\left(K_{n+1}\right)$ let $\mathscr{V}_{0} \leqq \mathscr{V}_{1} \leqq \ldots \leqq \mathscr{V}_{n+1}$ be a sequence of refinements as in 7.2. Now let $\mathscr{V}_{0}^{\prime}$ be such that $\left(\mathscr{V}_{0}, \mathscr{V}_{0}^{\prime}\right)$ has $\operatorname{ECR}\left(n_{0}\right)$ with respect to $\left(\mathscr{C}_{0}^{\prime}, \mathscr{C}_{0}^{\prime \prime}\right)$ and let $\tau$ be a partial realization of $S$ in $\Delta C\left(K, \mathscr{V}_{0}^{\prime}\right), \tau: S_{1} \rightarrow$ $\Delta C\left(K, \mathscr{V}_{0}^{\prime}\right)$ of norm $\leqq \mathscr{C}_{0}^{\prime \prime}$.

Then there exists an extension $\tau_{0}: S_{1} \cup S^{n_{0}} \rightarrow \Delta C\left(K_{0}, \mathscr{V}_{0}\right)$ of norm $\leqq \mathscr{C}_{0}^{\prime}$ which in turn can be extended to $\tau_{n+1}: S_{1} \cup S^{n_{0+n+1}} \rightarrow \Delta C\left(K_{n+1}, \mathscr{V}_{n+1}\right)$ by 7.3. This extension $\tau_{n+1}$ satisfies (7.3.2) and therefore is of norm $\leqq \mathscr{C}_{n+1}$.

7.9. Examples of $c-l c^{n}$ covers and refinements. Let $\mathscr{C}_{0}^{\prime}$ be any cover of $\mathbf{R}^{m}$ and $\mathscr{C}_{0}=\ldots=\mathscr{C}_{n}=\left\{\mathbf{R}^{m}\right\}$. Applying the corollary and 6.3 we see that any cover of $\mathbf{R}^{m}$ is a $c-l c^{n}$ cover for all $n$, i.e. a $c-l c^{\omega}$ cover.

Let $\mathscr{C}$ be a closed convex cover of $\mathrm{R}^{m}$ and $\mathscr{C}^{\prime} \leqq \mathscr{C}$ such that for every $C^{\prime} \in \mathscr{C}^{\prime}$ there is a $C \in \mathscr{C}$ with $C^{\prime} C$ interior $(C)$ then $\mathscr{C}^{\prime} \leqq \mathscr{C}$ is a $c-l c^{\omega}$ refinement.

7.10. Corollary. Let $\operatorname{dim} X \leqq n$ and let $\mathscr{C}^{\prime} \leqq \mathscr{C}$ be a $c-l c^{n}$ refinement where $\mathscr{C}$ is a closed cover. Let $\mathscr{C} \leqq \mathscr{C}^{\prime \prime}$ such that for every $C \in \mathscr{C}$ there is a $C^{\prime \prime} \in \mathscr{C}^{\prime \prime}$ with $C \subset$ interior $\left(C^{\prime \prime}\right)$. Then $\mathscr{C}^{\prime} \leqq \mathscr{C}^{\prime \prime}$ is a $c-l c^{\omega}$ refinement.

Proof. Take $\mathscr{C}_{0}=\ldots=\mathscr{C}_{n+1}=\mathscr{C}^{\prime \prime}$ in 7.8 . The acyclicity conditions now follow from the finite dimension assumption (cf. 3.4).

8. Acyclicity and $n$-geometric coverings. We can now construct $n$ geometric coverings given suitable acyclicity assumptions. 
8.1. Theorem. Let $\mathscr{U}^{\prime} \leqq \mathscr{C}_{0}^{\prime} \leqq \mathscr{C}_{0} \leqq \ldots \leqq \mathscr{C}_{n+1}$ be a sequence of covers of a space $X$ such that

(i) $\mathscr{U}^{\prime}$ is a finite open cover; $\mathscr{C}_{0}{ }^{\prime}, \mathscr{C}_{0}, \ldots, \mathscr{C}_{n+1}$ are finite closed covers;

(ii) for every $C_{0}{ }^{\prime} \in \mathscr{C}_{0}{ }^{\prime}$ there is a $C_{0} \in \mathscr{C}_{0}$ such that $C_{0}{ }^{\prime} \subset$ interior $\left(C_{0}\right)$;

(iii) $\mathscr{U}^{\prime}$ is a star refinement of $\mathscr{C}_{0}{ }^{\prime}$;

(iv) $\mathscr{C}_{k} \leqq \mathscr{C}_{k+1}$ is acyclic of dimension $k$.

Then $\mathscr{C}_{n+1}$ is n-geometric and $\mathscr{U}^{\prime} \in\left(\mathfrak{H}_{n+1}\right)$.

Proof. Let $\Gamma$ be any cofinal collection of compact subsets of $X$. For every $K \in \Gamma$ choose a sequence $K=K_{0} \subset K_{1} \subset \ldots \subset K_{n+1}$ such that (7.2.3) is satisfied. Enlarging $K_{n+1}$ if necessary we can assume that also $K_{n+1} \in \Gamma$. Define $\gamma K=K_{n+1}$. For every $\mathscr{V}=\mathscr{V}_{n+1} \in \operatorname{cov}\left(K_{n+1}\right)$ refining $\mathscr{U}^{\prime}$ choose a sequence

$$
\mathscr{V}_{0} \leqq \mathscr{V}_{1} \leqq \ldots \leqq \mathscr{V}_{n+1} \quad \mathscr{V}_{i} \in \operatorname{cov}^{f}\left(K_{i}\right)
$$

such that (7.2.5) is satisfied. For each $C_{0}{ }^{\prime}(i) \in \mathscr{C}_{0}{ }^{\prime}$ choose $C_{0}(i) \in \mathscr{C}_{0}$ such that $C_{0}{ }^{\prime}(i) \subset$ interior $\left(C_{0}(i)\right)$. Refining $\mathscr{V}_{0}$ if necessary we can assume that

(8.1.1) $V_{0} \in \mathscr{V}_{0}, \quad V_{0} \cap C_{0}{ }^{\prime}(i) \neq \emptyset \Rightarrow V_{0} \subset C_{0}(i)$.

Let $S=C\left(K, \mathscr{U}^{\prime}\right), S_{1}=S^{0}$. We now define $\tau_{0}: S^{0} \rightarrow C\left(K_{0}, \mathscr{V}_{0}\right)$ as follows. For each $U^{\prime}(i) \in \mathscr{U}^{\prime}$ choose a $V_{0}(i)$ such that $V_{0}(i) \cap U^{\prime}(i) \neq \emptyset$, and define $\tau_{0}\left(U^{\prime}(i)\right)=V_{0}(i)$. That is, we have

(8.1.2) $\tau_{0}\left(U^{\prime}(i)\right) \cap U^{\prime}(i) \cap K_{0} \neq \emptyset$.

For each $U^{\prime}(i)$ choose $C_{0}{ }^{\prime}(i)$ such that $\operatorname{star}\left(U^{\prime}(i)\right) \subset C_{0}{ }^{\prime}(i)$. That is,

(8.1.3) $U^{\prime}(j) \cap U^{\prime}(i) \neq \emptyset \Rightarrow U^{\prime}(j) \subset C_{0}{ }^{\prime}(i)$.

Now let $\sigma=\left\{U^{\prime}\left(i_{0}\right), \ldots, U^{\prime}\left(i_{m}\right)\right\}$ be a maximal simplex of $S=C\left(K, \mathscr{U}^{\prime}\right)$, i.e. $U^{\prime}\left(i_{0}\right) \cap \ldots \cap U^{\prime}\left(i_{m}\right) \cap K \neq \emptyset$ and hence

(8.1.4) $\quad U^{\prime}\left(i_{r}\right) \subset C_{0}{ }^{\prime}\left(i_{s}\right), \quad r, s=0,1, \ldots, m$.

It follows that

(8.1.5) $\operatorname{supp}(\sigma) \subset C_{0}{ }^{\prime}\left(i_{s}\right) \cap K, \quad s=0,1, \ldots, m$.

Now $\tau_{0}\left(U^{\prime}\left(i_{r}\right)\right) \cap U^{\prime}\left(i_{r}\right) \cap K_{0} \neq \phi, K_{0} \cap U^{\prime}\left(i_{r}\right) \subset C_{0}{ }^{\prime}\left(i_{s}\right) \cap K_{0}$. Hence

(8.1.6) $\tau_{0}\left(U^{\prime}\left(i_{r}\right)\right)$ is on $C_{0}{ }^{\prime}\left(i_{s}\right) \cap K_{0}, \quad r=0, \ldots, m ; s=0, \ldots, m$,

which by (8.1.1) implies

(8.1.7) $\tau_{0}\left(U^{\prime}\left(i_{\tau}\right)\right) \subset C_{0}\left(i_{s}\right) \cap K_{0}, \quad r, s=0, \ldots, m$.

We can now choose the $\mathscr{A}(\sigma)$ as follows. For each maximal simplex $\sigma_{t}$ choose a vertex $U^{\prime}(t)$ of $\sigma_{t}$ and let $C_{0}{ }^{\prime}\left(\sigma_{t}\right)=C_{0}{ }^{\prime}(t)$. Then (cf. 7.3) $\mathscr{A}(\sigma)=$ $\left\{C_{0}{ }^{\prime}\left(\sigma_{t}\right) \mid \sigma \subset \sigma_{t}, \sigma_{t}\right.$ maximal $\}$. In view of $(8.1 .5),(8.1 .7)$ we have for all 
$\sigma \in C\left(K, \mathscr{U}^{\prime}\right)$ and $\mu \in C^{0}\left(K, \mathscr{U}^{\prime}\right)$

(8.1.8) $\operatorname{supp}(\sigma) \subset(\cap \mathscr{A}(\sigma)) \cap K, \quad \operatorname{supp}\left(\tau_{0}(\mu)\right) \subset(\cap \mathscr{A}(\mu)) \cap K_{0}$.

Now, using these $\mathscr{A}(\sigma)$, construct a sequence of extensions $\tau_{0}, \tau_{1}, \ldots, \tau_{n+1}$ as in (7.3). This gives chain maps

$$
\tau_{\mathscr{V}}=\tau_{n+1}: C^{r+1}\left(K, \mathscr{U}^{\prime}\right) \rightarrow \Delta C(\gamma K, \mathscr{V})
$$

We now check the various axioms which the $\tau_{\mathscr{r}}$ have to satisfy.

(a) The norm condition (cf. (3.1.4). This is satisfied because $\tau_{n+1}=\tau_{\mathscr{r}}$ satisfies (7.3.2) and because we have (8.1.8) (first part).

(b) The factorization property (cf. 3.1.2). Let $i: C\left(K, \mathscr{V}_{0}\right) \rightarrow C\left(K, \mathscr{U}^{\prime}\right)$ be any refining map; let $i_{k}: C\left(K_{k}, \mathscr{V}_{k}\right) \rightarrow C\left(K_{k+1}, \mathscr{V}_{k+1}\right)$ be refining maps for $k=0,1, \ldots, n$. Define $\bar{\tau}_{k}=\tau_{k} \circ i, k=0, \ldots, n+1$ and $\tau_{0}^{\prime}=\mathrm{id}$, $\tau_{k+1}{ }^{\prime}=i_{k} \circ \ldots \circ i_{0}, k=0, \ldots, n$. We then have two sequences of maps

$$
\bar{\tau}_{k}, \tau_{k}{ }^{\prime}: C\left(K, \mathscr{V}_{0}\right) \rightarrow C\left(K_{k}, \mathscr{V}_{k}\right)
$$

satisfying (7.3.1) and (7.3.2). Hence $H_{m}\left(\tau_{n+1}\right) . H_{m}(i)=H_{m}\left(\tau_{n+1}{ }^{\prime}\right), m=0$, $\ldots, n$ which proves the factorization property with $\mathscr{V}^{\prime}=\mathscr{V}_{0}$.

(c) The compatibility property (cf. 3.1.3). Let $\mathscr{V}_{1}, \mathscr{V}_{2}$ be two open covers of $\gamma K=K_{n+1}$ such that $\mathscr{V}_{1} \leqq \mathscr{V}_{2} \leqq \mathscr{U}^{\prime}$ let $\mathscr{V}_{0}(1), \ldots, \mathscr{V}_{n+1}(1)=\mathscr{V}_{1}$; $\mathscr{V}_{0}(2), \ldots, \mathscr{V}_{n+1}(2)=\mathscr{V}_{2}$ and let $\tau_{0}(1), \ldots, \tau_{n+1}(1)=\tau_{\mathscr{V}_{1}} ; \tau_{0}(2), \ldots$, $\tau_{n+1}(2)=\tau_{\mathscr{V}_{2}}$ be the corresponding sequences of chain maps. Choose a common refinement $\mathscr{V}_{0}$ of $\mathscr{V}_{0}(1)$ and $\mathscr{V}_{0}(2)$. Define $\tau_{0}: C^{0}\left(K, \mathscr{U}^{\prime}\right) \rightarrow \Delta C\left(K_{0}, \mathscr{V}_{0}\right)$ by assigning to each $U^{\prime}$ a $V_{0}$ such that $U^{\prime} \cap V_{0} \neq \emptyset$. Let $i_{1}: \mathscr{V}_{0} \rightarrow \mathscr{V}_{0}(1)$ (resp. $i_{2}: \mathscr{V}_{0} \rightarrow \mathscr{V}_{0}(2)$ ) be any refinement map. Define $\tau_{0}{ }^{\prime}(1)=i_{1} \tau_{0}$, $\tau_{0}{ }^{\prime}(2)=i_{2} \tau_{0}$ and let $\tau_{0}{ }^{\prime}(1), \ldots, \tau_{n+1}{ }^{\prime}(1)$ (resp. $\tau_{0}{ }^{\prime}(2), \ldots, \tau_{n+1}{ }^{\prime}(2)$ ) be the sequences of extensions obtained by using $\mathscr{V}_{0}(1) \leqq \ldots \leqq \mathscr{V}_{n+1}(1)$ (resp. $\left.\mathscr{V}_{0}(2) \leqq \ldots \leqq \mathscr{V}_{n+1}(2)\right)$. Then $H_{m}\left(\tau_{n+1}{ }^{\prime}(1)\right)=H_{m}\left(\tau_{n+1}(1)\right)$ and $H_{m}\left(\tau_{n+1}{ }^{\prime}(2)\right)=H_{m}\left(\tau_{n+1}(2)\right)$ for $m=0, \ldots, n$. The sequences

$$
\tau_{0}, \tau_{1}^{\prime}(1), \ldots, \tau_{n}{ }^{\prime}(1), i \circ \tau_{n+1}{ }^{\prime}(1) ; \tau_{0}, \tau_{1}{ }^{\prime}(2), \ldots, \tau_{n+1}{ }^{\prime}(2)
$$

where $i$ is any refinement map $C\left(\gamma K, \mathscr{V}_{1}\right) \rightarrow C\left(\gamma K, \mathscr{V}_{2}\right)$, are sequences of extensions corresponding to the sequences of refinements

$$
\mathscr{V}_{0}, \mathscr{V}_{1}(1), \ldots, \mathscr{V}_{n}(1), \mathscr{V}_{2} ; \mathscr{V}_{0}, \mathscr{V}_{1}(2), \ldots, \mathscr{V}_{n}(2), \mathscr{V}_{2}
$$

and therefore we have that $H_{m}\left(i_{\tau_{n+1}}{ }^{\prime}(1)\right)=H_{m}\left(\tau_{n+1}{ }^{\prime}(2)\right), m=0, \ldots, n$ and hence $H_{m}\left(i \tau_{n+1}(1)\right)=H_{m}\left(\tau_{n+1}(2)\right)$ for $m=0,1, \ldots, n$, which proves the compatibility.

This theorem is especially useful in the case of convex or more generally convexoid covers.

8.2. Convexoid covers (definition). A finite closed cover $\mathscr{C}$ of a space $X$ is called convexoid of dimension $\leqq n$, if $\mathscr{C} \leqq \mathscr{C}$ is acylic of dimension $k$ for all $k=0,1, \ldots, n$. That is, for every $k=0,1, \ldots, n$ and for every subset 
$\mathscr{A} \subset \mathscr{C}$ and every compact set $K \subset \cap \mathscr{A}$ there is a larger compact set $K^{\prime} \subset \cap \mathscr{A}, K \subset K^{\prime}$ such that $\widetilde{H}_{k}(K) \rightarrow \widetilde{H}_{k}\left(K^{\prime}\right)$ is the zero map. $\mathscr{C}$ is called convexoid if it is convexoid of dimension $\leqq n$ for all $n \in \mathrm{N}$.

8.3. Corollary. Let $\mathscr{U}^{\prime} \leqq \mathscr{C}^{\prime} \leqq \mathscr{C}$ be a sequence of covers such that

(i) $\mathscr{U}^{\prime}$ is finite open, $\mathscr{C}^{\prime}, \mathscr{C}$ are finite clostd covers;

(ii) $\mathscr{U}^{\prime}$ is a star refinement of $\mathscr{C}^{\prime}$;

(iii) for every $C^{\prime} \in \mathscr{C}^{\prime}$ there is a $C \in \mathscr{C}$ such that $C^{\prime} \subset$ interior $(C)$;

(iv) $\mathscr{C}$ is n-convexoid.

Then $\mathscr{C}$ is n-geometric and $\mathscr{U}^{\prime} \in \mathfrak{U}(\mathscr{C})$.

Proof. Apply 8.1 with $\mathscr{C}_{0}^{\prime}=\mathscr{C}, \mathscr{C}_{0}=\mathscr{C}_{1}=\ldots=\mathscr{C}_{n+1}=\mathscr{C}$.

8.4. THEOREM. Let $\mathscr{C}$ be a finite closed convexoid covering of a normal space $X$ which admits a finite open refinement. Then $\mathscr{C}$ is geometric.

Proof. Let $\mathscr{U}$ be a finite open refinement of $\mathscr{C}$. Because $X$ is normal there exists a finite open star refinement $\mathscr{V}$ of $\mathscr{U}$. Let $\mathscr{C}^{\prime}$ be the covering consisting of the closures of elements of $\mathscr{V}$. Finally let $\mathscr{U}^{\prime}$ be a finite open star refinement of $\mathscr{V}$. The chain of coverings

$$
\mathscr{U}^{\prime} \leqq \mathscr{C}^{\prime} \leqq \mathscr{C}_{0} \leqq \ldots \leqq \mathscr{C}_{n+1}
$$

with $\mathscr{C}=\mathscr{C}_{0}=\mathscr{C}_{1}=\ldots=\mathscr{C}_{n+1}$ then satisfies the conditions of Theorem 8.1.

8.5. Further remark. Instead of relying on acyclicity conditions to construct geometric covers one can also rely on $c-l c^{n}$ refinements in order to be able to construct the necessary chain maps. In fact for the application to the construction of $n$-geometric covers a somewhat weaker notion: weak $c-l c^{n}$ refinement is sufficient. This is defined as follows.

Let $K \subset K^{\prime}, \mathscr{V} \in \operatorname{cov}^{f}(K), \mathscr{V}^{\prime} \in \operatorname{cov}^{f}\left(K^{\prime}\right)$. The pair $\left(\mathscr{V}^{\prime}, \mathscr{V}\right)$ is said to have weak $E C R(n)$ with respect to $\left(\mathscr{C}^{\prime}, \mathscr{C}\right)$ if for every complex $S$ of dimension $\leqq n$ and partial realization $\tau: S_{1} \rightarrow \Delta C(K, \mathscr{V})$ of norm $\leqq \mathscr{C}$ there exists a realization $\tau^{\prime}: S \rightarrow \Delta C\left(K^{\prime}, \mathscr{V}^{\prime}\right)$ of norm $\leqq \mathscr{C}^{\prime}$ extending $\tau$.

A pair of covers $\mathscr{C}_{1} \leqq \mathscr{C}_{2}$ of a space $X$ is then a weak $c-l c^{n}$ refinement if for every compact $K_{1} \subset X$ there is a larger compact $K_{2}$ such that for every $\mathscr{V}_{2} \in \operatorname{cov}^{f}\left(K_{2}\right)$ there is a refinement $\mathscr{V}_{1} \in \operatorname{cov}^{f}\left(K_{1}\right)$ such that $\left(\mathscr{V}_{2}, \mathscr{V}_{1}\right)$ has weak $\operatorname{ECR}(n+1)$ with respect to $\left(\mathscr{C}_{2}, \mathscr{C}_{1}\right)$.

One now has, for example, the following theorem.

THEOREM. Suppose we have a sequence of covers $\mathscr{U}^{\prime} \leqq \mathscr{C}^{\prime} \leqq \mathscr{C}^{\prime \prime} \leqq \mathscr{C}$ of a space $X$ such that

(i) $\mathscr{U}^{\prime}$ is a finite open cover; ' $\mathscr{C}^{\prime}, \mathscr{C}^{\prime \prime}, \mathscr{C}$ ure finite closed covers,

(ii) $\mathscr{U}^{\prime}$ is a star refinement of $\mathscr{C}^{\prime}$; $\mathscr{C}$ " is a stur refinement of $\mathscr{C}$;

(iii) $\mathscr{C}$ is a weak $c-l c^{n}$ covering of $X$ (i.e. $\mathscr{C} \leqq\{X\}$ is a weak $c-l c^{n}$ refinement.

Suppose that in addition one of the following conditions is sutisfied: 
(iv) $\mathscr{C}^{\prime}$ is a weak $c-l c^{n}$ refinement of $\mathscr{C}^{\prime \prime}$;

(v) $\operatorname{dim} \mathscr{U}^{\prime} \leqq n$ and $\mathscr{C}^{\prime}$ is a weak $c-l c^{n-1}$ refinement of $\mathscr{C}^{\prime \prime}$;

(vi) $\operatorname{dim} X \leqq n$ and $\mathscr{C}^{\prime}$ is a $c-l c^{n-1}$ refinement of $\mathscr{C}^{\prime \prime}$.

Then $\mathscr{C}$ is n-geometric and $\mathscr{U}^{\prime} \in \mathfrak{A}(\mathscr{C})$.

The proof of this theorem is very similar to the proof of Theorem 8.1. However, one has slightly weaker control of the supports and it is to overcome this that one needs the extra starrefinement $\mathscr{C}^{\prime \prime} \leqq \mathscr{C}$ and condition (iii). For detail cf. [15].

9. Convex covers of topological vector spaces. In order to prove that vector spaces have the almost fixed point property with respect to finite open convex covers and continuous maps we need the following refinement-ofconvex-coverings result.

9.1. Theorem. Let $A$ be a finite intersection of closed halfspaces in $\mathbf{R}^{n}$ or $A=$ $\mathbf{R}^{n}$. Then for every finite open convex covering $\mathscr{U}$ of $A$ there exists a finite closed convex covering $\mathscr{C}$ such that

(i) $\mathscr{C} \leqq \mathscr{U}$, i.e. $\mathscr{C}$ refines $\mathscr{U}$; and

(ii) the interiors of the elements of $\mathscr{C}$ still cover $A$.

To prove this we use some lemmas. Let $U \subset \mathbf{R}^{n}, U \neq \mathbf{R}^{n}$ be a convex set, $U$ its closure. We define a function $r_{U}: \bar{U} \rightarrow \mathbf{R}$ by $r_{U}(x)=\sup \{r \mid B(x, r) \subset$ $U\}$ where $B(x, r)$ is the open ball of radius $r$ and center $x$.

9.2. Lemma. $r_{U}: \bar{U} \rightarrow \mathbf{R}$ is a concave continuous function, that is,

$$
r_{U}(\lambda x+(1-\lambda) y) \geqq \lambda_{U}(x)+(1-\lambda) r_{U}(y) \text { for } x, y \in \bar{U} .
$$

9.3. Lemma. Let $C$ be a closed convex subset of an open convex set $U \subset \mathbf{R}^{n}$. Then there is a closed convex subset $C^{\prime}$ such that $C \subset \operatorname{int}\left(C^{\prime}\right) \subset C^{\prime} \subset U$.

Proof. Define

$$
C^{\prime \prime}=\left\{x \in \bar{U} \mid \text { there exists } y \in C \text { such that }\|x-y\| \leqq \frac{1}{2} r_{U}(y)\right\} .
$$

We check that $C^{\prime \prime}$ is convex. Let $\left\|x_{1}-y_{1}\right\| \leqq \frac{1}{2} r_{U}\left(y_{1}\right),\left\|x_{2}-y_{2}\right\| \leqq \frac{1}{2} r_{U}\left(y_{2}\right)$. Let $0 \leqq \lambda \leqq 1$. Then

$$
\begin{aligned}
& \|\left(\lambda x_{1}+(1-\lambda) x_{2}\right)-\left(\lambda y_{1}+(1-\lambda) y_{2} \|\right. \leqq \lambda\left\|x_{1}-y_{1}\right\| \\
&+(1-\lambda)\left\|x_{2}-y_{2}\right\| \leqq \frac{1}{2} \lambda r_{U}\left(y_{1}\right)+\frac{1}{2}(1-\lambda) r_{U}\left(y_{2}\right) \\
& \\
& \leqq \frac{1}{2} r_{U}\left(\lambda y_{1}+(1-\lambda) y_{2}\right)
\end{aligned}
$$

because $r_{U}$ is concave. Now let $x$ be a point in the closure of $C^{\prime \prime}$. We show that $x \in U$. This will prove the lemma. (Take $C^{\prime}=\bar{C}^{\prime \prime}$ ). Let $\left(x_{i}\right) \in C^{\prime \prime}$ be a sequence of points converging to $x$. Let $y_{i}$ be such that $\left\|x_{i}-y_{i}\right\| \leqq \frac{1}{2} r_{U}\left(y_{i}\right)$. Note that $r_{U}\left(x_{i}\right) \geqq \frac{1}{2} r_{U}\left(y_{i}\right)$. Consider the sequence of real positive numbers $r_{U}\left(y_{i}\right)$. If $\lim \inf r_{U}\left(y_{i}\right)>0$ then lim inf $r_{U}\left(x_{i}\right)>0$ and hence $r_{U}(x)>0 \Rightarrow x \in U$. If $\lim \inf r_{U}\left(y_{i}\right)=0$ we can assume by taking a subsequence that $\lim r_{U}\left(y_{i}\right)=0$ 
because $\left\|x_{i}-y_{i}\right\| \leqq \frac{1}{2} r_{U}\left(y_{i}\right)$ it follows that $y_{i}$ converges to $x$. But $y_{i} \in C$ and $C$ is closed hence $x \in C \subset U$.

9.4. Lemma. Let $A \subset \mathbf{R}^{n}$ be a convex set and let $x$ be a point in the interior of $A$. Let $l$ be a ray starting in $x$ and suppose $l \subset A$. Then

(i) $l \subset \operatorname{int}(A)$;

(ii) there exists $\epsilon>0$ such that $d(y, l) \leqq \epsilon \Rightarrow y \in \operatorname{int}(A)$, where $d(y, l)$ is the distance from $y$ to $l$.

Proof. Let $y \in l$, and let $y^{\prime}$ be a point on $l$ twice as far from $x$ as $y$. Let $B$ be small open ball around $x$ such that $B \subset A$. The linear combinations $\frac{1}{2} x+\frac{1}{2} y^{\prime}$, $y^{\prime} \in B$ then constitute an open ball around $y$, which proves that $y \in \operatorname{int}(A)$. This proves (i). To prove (ii) consider the function $r_{A}: l \rightarrow \mathbf{R}$ this function is concave and continuous and $r_{A}(y)>0$ for all $y \in l$. It follows that there is an $\epsilon>0$ such that $r_{A}(y) \geqq \epsilon$ for all $y \in l$. This proves (ii).

9.5. Proof of Theorem 9.1. If $\mathscr{U}$ is a finite open covering of $A$ we denote with $s(\mathscr{U})$ the total number of simplices in $C(\mathscr{U})$. By induction we can assume that the theorem has been proved for $\operatorname{dim} A<n$, and, $\operatorname{dim} A=n$ and $s(\mathscr{U})<s$. (The cases $\operatorname{dim} A=1$ and $s(\mathscr{U})=1$ being trivial.) Let $\mathscr{U}$ be a convex open covering of $A, \operatorname{dim} A=n$ and $s(\mathscr{U})=s$.

There are two cases to consider:

$$
\begin{aligned}
& \text { (a) } \cap \mathscr{U}=\emptyset \text {. } \\
& \text { (b) } \cap \mathscr{U} \neq \emptyset \text {. }
\end{aligned}
$$

In case (a) let $\mathscr{U}^{\prime} \subset \mathscr{U}$ be a maximal subset such that $\cap \mathscr{U}^{\prime} \neq \emptyset$. Choose $U \in \mathscr{U} \backslash \mathscr{U}^{\prime}$. By the separating hyperplane theorem there is a hyperplane $H$ such that $U$ is on one side of $H$ and $\cap \mathscr{U}^{\prime}$ on the other. Let $A^{+}$and $A^{-}$be the intersections of $A$ with the closed halfspaces determined by $H$. Then

$$
s\left(\mathscr{U} \mid A^{+}\right)<s(\mathscr{U}) \text { and } s\left(\mathscr{U} \mid A^{-}\right)<s(\mathscr{U})
$$

and by induction we are done with this case.

Suppose we are in case (b). We can assume that $A \subset \mathbf{R}^{n}, \operatorname{dim} A=n$ hence $\operatorname{int}(A) \neq \phi, \operatorname{dim} U=n$ for all $U \in \mathscr{U}$. Let $x \in \cap \mathscr{U}$. We can see to it that also $x \in \operatorname{int}(A)$. Let $S$ be a sphere with center $x$. Each point $s \in S$ corresponds uniquely to a ray $l_{s}$ starting in $x$. For every ray $l_{s}$ there is a $U \in \mathscr{U}$ such that $l_{s} \subset U$. This is seen as follows. If $l_{s} \cap A \neq l_{s}$ then there is a unique point $y_{s} \in l_{s} \cap A$ such that $l_{s} \cap A=$ segment joining $x$ and $y_{s} ; y_{s}$ is in the boundary of $A$. If $l_{s} \cap A=l_{s}$ choose points $y_{1}, y_{2}, y_{3}, \ldots$ on $l_{s}$ at distance $1,2,3, \ldots$ from $x$. At least one $U \in \mathscr{U}$ contains infinitely many of these points. Then $l_{s} \subset U$.

For every $U \in \mathscr{U}$ we now define a set $C_{U}$ as follows:

$$
C_{U}=\left\{y \in A \mid \text { there exists } s \in S \text { such that } y \in l_{s} \subset U\right\} .
$$

Concerning these $C_{U}$ we have: 
(i) $C_{U}$ is convex, $C_{U} \subset U$;

(ii) $C_{U}$ is closed.

Claim (i) is a triviality. To prove (ii), consider $C_{U} \cap S$. Let $\left(s_{i}\right), s_{i} \in C_{U} \cap S$ be a sequence of points converging to $s \in S$. Let $z$ be a point of $l_{s}$. Then $z$ is the limit of a sequence of points $z_{i} \in l_{s_{i}}$ (take $z_{i} \in l_{s i}$ such that $\left\|z_{i}-x\right\|=$ $\|z-x\|$. Hence $z \in \bar{U}$. Hence $l_{s} \subset U$ and hence $l_{s} \subset \operatorname{int}(\bar{U})$ by Lemma 9.4. This proves (ii).

To deal with the rays $l_{s}$ such that $l_{s} \cap A \neq l_{s}$ we use the following construction. The boundary $\operatorname{bd}(A)$ of $A$ is a finite union $A=\bigcup_{t=1}^{k} A_{t}, \operatorname{dim}$ $A_{t}=n-1, A_{t}$ a finite intersection of closed halfspaces. For each $t$ let $D_{1}(t), \ldots, D_{n t}(t)$ be a finite closed convex covering of $A_{t}$ which refines $\mathscr{U} \mid A_{t}$. (Induction!)

For each $t$ and $i \in\left\{1, \ldots, n_{t}\right\}$ we define

$$
C_{t, i}=\left\{y \in A \mid \text { there exists } x \in D_{i}(t) \text { with } y\right. \text { on the sequent joing }
$$

$x$ and $z\}$.

For each $C_{t, i}$ choose $U \in \mathscr{U}$ such that $D_{i}(t) \subset U$. Then we have

(iii) $C_{t, i} \subset U$ and $C_{t, i}$ is convex;

(iv) $\bar{C}_{t, i} \subset U$.

Claim (iii) is a triviality. To see (iv) let $T \subset S$ be the subset of $s \in S$ corresponding to rays in $C_{t, i}$. Let $\left(s_{i}\right), s_{i} \in T$ be a sequence of points converging to $s \in S$. There are two possibilities. First $l_{s} \cap A \neq l_{s}$. The sequence $y_{s i}$ of endpoints then converges to $y_{s}$ and because $y_{s i} \in D_{i}(t), y_{s} \in D_{i}(t)$ so that $l_{s} \cap A \subset C_{t, i} \subset U$. Secondly suppose that $l_{s} \cap A=l_{s}$ (i.e. the points $y_{s i}$ run off to infinity). Let $z \in l_{s}$. The distance $\left\|x-y_{s i}\right\|$ goes to infinity as $i \rightarrow \infty$ (this follows from Lemma 9.4 (ii)). Hence $z$ is the limit of a sequence of points $z_{i} \in l_{s i} \cap A \subset U$. Hence $z \in \bar{U}$. Thus $l_{s} \subset \bar{U}$ hence $l_{s} \subset \bar{U}$ by Lemma 9.4. (i). We have now found a closed convex finite covering consisting of

$$
C_{U}, U \in \mathscr{U} ; \quad \bar{C}_{t, i}, t=1, \ldots, k ; i=1, \ldots, n_{t}
$$

of $A$ which refines $\mathscr{U}$. Thickening each $C_{U}$ and $\bar{C}_{t, i}$ as in Lemma 9.3 then gives a finite closed convex covering $\mathscr{C}$ which refines $\mathscr{U}$ and such that their interiors still cover $A$. This concludes the proof of the theorem.

We now use Theorem 9.1 to obtain the following generalization:

9.6. Theorem. Let $X$ be a locally convex linear space and let $\mathscr{U}$ be a finite convex open cover of $X$. Then there exists a finite convex closed cover $\mathscr{C}$ of $X$ refining $\mathscr{U}$, such that the interiors of the elements of $\mathscr{C}$ still cover $X$.

Proof. Since $\mathscr{U}$ is finite, there is a minimal subcollection $\mathscr{U}_{0} \subset \mathscr{U}$ covering $X$. This cover obviously satisfies the property below:

Each member $U$ of $\mathscr{U}_{0}$ contains a point $x_{U}$ which is not covered by the other members of $\mathscr{U}_{0}$. We may assume $\mathscr{U}=\mathscr{U}_{0}$ and we fix $x_{U} \in U$ as above for each $U \in \mathscr{U}$. 
If $U^{\prime} \in \mathscr{U}-\{U\}$ then $x_{U} \notin U^{\prime}$ and applying a Hahn-Banach theorem, there is a continious linear functional

$$
f_{U^{\prime}}{ }^{U}: X \rightarrow \mathbf{R}
$$

such that $f_{U^{\prime}}{ }^{U}(x)<1 \leqq f_{U^{\prime}}{ }^{U}\left(x_{U}\right)$ for each $x \in U^{\prime}$. Then

$$
M_{U}=\bigcap_{U^{\prime} \neq U} \operatorname{ker} f_{U^{\prime}}{ }^{U} \quad(U \text { fixed })
$$

is a closed vectorsubspace of finite codimension. The coset of $M_{U}$, determined by $x_{U}$ is denoted by $x_{U}+M_{U}$. Then $f_{U^{\prime}}{ }^{U}$ takes the constant value $f_{U^{\prime}}{ }^{U}\left(x_{U}\right) \geqq 1$ on $x_{U}+M_{U}$, and hence

$$
\left(x_{U}+M_{U}\right) \cap U^{\prime}=\phi, \quad U^{\prime} \neq U,
$$

proving that

$$
x_{U}+M_{U} \subset U .
$$

Next define

$$
M=\bigcap_{U \in \mathscr{\ell}} M_{U}
$$

$M$ is again a closed subspace of finite codimension and $x_{U}+M \subset U$ for all $U \in \mathscr{U}$. It easily follows that $x+M \subset U$ whenever $x \in U$, and hence each $U \in \mathscr{U}$ is saturated with respect to the linear identification map

$$
\pi: X \rightarrow X / M \text {. }
$$

Now $X / M$ is a finite dimensional linear space which is therefore isomorphic to some euclidean space, $\mathbf{R}^{n}$ say. By definition of the quotient topology, $\pi(U) \subset$ $\mathbf{R}^{n}$ is an open set, which is easily seen to be convex. Applying theorem 9.1 on the finite convex open cover $\{\pi(U) \mid U \in \mathscr{U}\}$ of $\mathbf{R}^{n}$ we find a finite convex closed refinement $\mathscr{D}$ of $\mathscr{U}$ whose interiors still cover $\mathbf{R}^{n}$. Then $\mathscr{C}=\pi^{-1}(\mathscr{D})$ is a finite convex closed cover of $X$ refining $\mathscr{U}$, and the in teriors of $\mathscr{C}$ still cover $X$, since for $C \in \mathscr{C}$,

$$
\text { interior } \pi^{-1}(C) \supset \pi^{-1} \text { (interior } C \text { ). }
$$

We can now prove the following almost fixed point property for locally convex linear spaces, which was conjectured by De Groot for eucliclean spaces, cf. [3].

9.7. Almost fixed point theorem for locally convex spaces.

THEOREM. Let $\mathscr{U}$ be a finite convex open covering of a locally convex space $X$ and let $f: X \rightarrow X$ be a continuous map. Then there is $U \in \mathscr{U}$ such that $U \cap f(U)$ $\neq \emptyset$.

Proof. We construct a sequence of refinements

$$
\mathscr{U}_{2} \leqq \mathscr{C}_{2} \leqq \mathscr{U}_{1} \leqq \mathscr{C}_{1} \leqq \mathscr{U}_{0} \leqq \mathscr{C}_{0} \leqq \mathscr{U}
$$


as follows. Let $\mathscr{C}_{0}$ be a finite convex closed refinement of $\mathscr{U}$ such that the collection $\mathscr{U}_{0}$ of interiors of members of $\mathscr{C}_{0}$ still cover $X$. Then $\mathscr{U}_{0}$ is again a finite convex open cover, and we repeat this process twice to get the other members of the above sequence. Since $\mathscr{C}_{2}$ is closed, Lemma 5.2 applies. There is a finite open cover $\mathscr{V}$ of $X$ (not necessarily convex) and a refining map $\alpha$ : $\mathscr{C}_{2} \rightarrow \mathscr{U}_{1}$ such that each $V \in \mathscr{V}$ intersecting $C_{2} \in \mathscr{C}_{2}$ is contained in $\alpha\left(C_{2}\right) \in \mathscr{U}_{1}$.

Let $\mathscr{U}^{\prime}$ be a common refinement of $\mathscr{U}_{2}$ and $\mathscr{V}$. Then $\mathscr{U}^{\prime}$ is a star refinement of $\mathscr{U}_{1}$ and hence of $\mathscr{C}_{1}$. We can now apply Corollary 8.3 with $\mathscr{C}^{\prime}=\mathscr{C}_{1}$, $\mathscr{C}=\mathscr{C}_{0}$, showing that $\mathscr{C}_{0}$ is geometric. Finally, apply Theorem 5.3, using the fact that $\tilde{H}(X)=0$ and hence $L(f) \neq 0$.

\section{REFERENCES}

1. E. G. Begle, Locally connected spaces and generalized manifolds, Amer. J. Math. 64 (1942), .5.53-574.

2. J. Dugundji, A duality property of neries, Fund. Math. 59 (1966), 213-219.

3. J. de Groot, H. de Vries and T. van der Walt, Almost fixed point theorems for the Euclidean plane. Proc. Nederl. Akad. Wetenschappen Ser A 66 (1963), 606-612.

4. S. Eilenberg and N. Steenrod, Foundations of algebraic topology (Princeton Univ. Press, $1952)$.

5. S. Eilenberg and D. Montgomery, Fixed point theorems for multicalued transformations, Amer. J. Math. 68 (1946), 214-222.

6. W. J. Gray and L. Vaughan, The almost fixed point property for hereditary unicoherent continua, Proc. Amer. Math. Soc. 27 (1971), 381-386.

7. B. Halpern, Almost fixed points for subsets of $\mathbf{Z}^{n}$, J. Combinatorial Theory Ser. A 11 (1971), $251-257$.

8. V. Klee, Some topological properties of coniex sets, Trans. Amer. Math. Soc. 78 (1955), $30-45$.

9. S. Lefschetz, Algebraic topology (American Math. Soc., 1942).

10. - Topics in topology (Princeton Univ. Press, 1942).

11. E. Spanier, Algebraic topology (McGraw-Hill, 1966).

12. R. B. Thompson, A unified approach to local and global fixed point indices, Advances Math. 3 (1969), 1-71.

13. - - Fixed point theory s'ia semicomplexes, Rocky Mountain J. Math. 4 (1974).

14. - Fixed point indices in locally convex spaces, Advances Math. 14 (1974), 73-91.

15. M. Van de Vel, The intersection property: A contribution to almost fixed point theory, Thesis, Univ. Instelling Antwerpen, 1975.

16. T. Van der Walt, Fixed and almost fixed points, Thesis, Math. Centre, Amsterdam, 1963.

Erasmus University of Rotterdam,

Rotterdam, The Netherlands;

Free University of Amsterdam,

Amsterdam, The Netherlands 